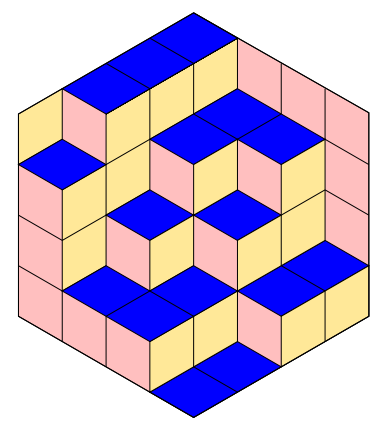

ALGEBRAIC COMBINATORICS

Sara C. Billey, Alexander E. Holroyd \& Benjamin J. Young A bijective proof of Macdonald's reduced word formula Volume 2, issue 2 (2019), p. 217-248.

<http://alco.centre-mersenne.org/item/ALCO_2019__2_2_217_0>

(c) The journal and the authors, 2019.

Some rights reserved.

\begin{tabular}{|l|l}
\hline (c) BY This article is licensed under the \\
\hline
\end{tabular}

Creative Commons ATtribution 4.0 InTERnational License.

http://creativecommons.org/licenses/by/4.0/

Access to articles published by the journal Algebraic Combinatorics on the website http://alco.centre-mersenne.org/ implies agreement with the Terms of Use (http://alco.centre-mersenne.org/legal/).

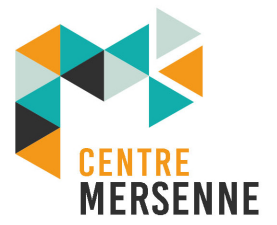

Algebraic Combinatorics is member of the Centre Mersenne for Open Scientific Publishing www.centre-mersenne.org 


\title{
A bijective proof of Macdonald's reduced word formula
}

\author{
Sara C. Billey, Alexander E. Holroyd \& Benjamin J. Young
}

\begin{abstract}
We give a bijective proof of Macdonald's reduced word identity using pipe dreams and Little's bumping algorithm. This proof extends to a principal specialization due to Fomin and Stanley. Such a proof has been sought for over 20 years. Our bijective tools also allow us to solve a problem posed by Fomin and Kirillov from 1997 using work of Wachs, Lenart, Serrano and Stump. These results extend earlier work by the third author on a Markov process for reduced words of the longest permutation.
\end{abstract}

\section{INTRODUCTION}

Macdonald gave a remarkable formula connecting a weighted sum of reduced words for a permutation $\pi$ with the number of terms in a Schubert polynomial $\mathfrak{S}_{\pi}\left(x_{1}, \ldots, x_{n}\right)$. For a permutation $\pi \in S_{n}$, let $\ell(\pi)$ be its inversion number and let $R(\pi)$ denote the set of its reduced words. (See Section 2 for definitions.)

THEOREM 1.1 (Macdonald $[33,(6.11)]$ ). Given a permutation $\pi \in S_{n}$ with $\ell(\pi)=p$, one has

$$
\sum_{\left(a_{1}, a_{2}, \ldots, a_{p}\right) \in R(\pi)} a_{1} \cdot a_{2} \cdots a_{p}=p ! \mathfrak{S}_{\pi}(1, \ldots, 1) .
$$

For example, the permutation $[3,2,1] \in S_{3}$ has 2 reduced words, $R([3,2,1])=$ $\{(1,2,1),(2,1,2)\}$. The inversion number is $\ell([3,2,1])=3$, and the Schubert polynomial $\mathfrak{S}_{\pi}\left(x_{1}, x_{2}, x_{3}\right)$ is the single term $x_{1}^{2} x_{2}$. We observe that Macdonald's formula holds: $1 \cdot 2 \cdot 1+2 \cdot 1 \cdot 2=3$ ! $\cdot 1$.

In this paper, we give a bijective proof of Theorem 1.1. Such a proof has been sought for over 20 years. It has been listed as an open problem in both [11] and [45]. Fomin and Sagan have stated that they have a bijective proof, but that it is unpublished due to its complicated nature; see [11]. Moreover, we give several generalizations as discussed below. Our proof builds on the work of the third author on a Markov process on reduced words for the longest permutation [49].

The Schubert polynomial $\mathfrak{S}_{\pi}$ can be expressed as a sum over reduced pipe dreams (or $R C$ graphs) corresponding to $\pi$, and its evaluation at $(1, \ldots, 1)$ is simply the number of such pipe dreams. (See Section 2 for definitions, and $[27,3,12,10,1]$ for history and proofs.) Thus, the right side of (1) is the number of pairs $(\mathbf{c}, D)$, where $\mathbf{c}=\left(c_{1}, \ldots, c_{p}\right)$ is a word with $1 \leqslant c_{i} \leqslant i$ for each $i$, and $D$ is a pipe dream for $\pi$.

Manuscript received 30th August 2017, accepted 29th May 2018.

ACKNowledgements. Billey was partially supported by grant DMS-1101017 from the NSF. 
a:

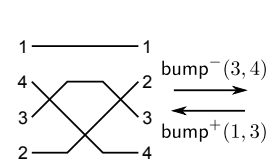

b:

212

$\downarrow M$

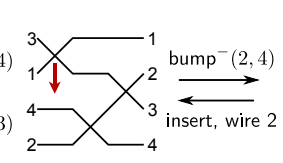

112

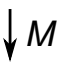

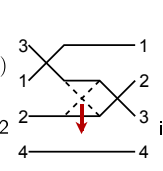

$1-2$

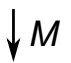

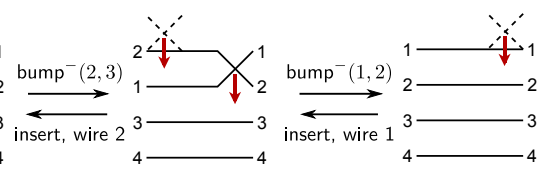

c: $\quad 112$

112

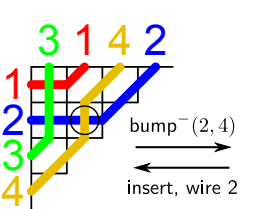

11
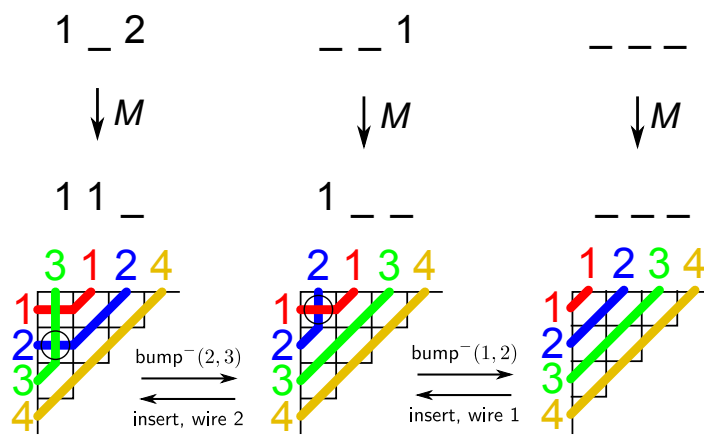

1234
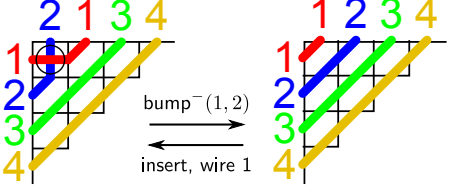

Figure 1. An example of the bijection $M$ for $\pi=[1,4,3,2]$ where the pair $(\mathbf{a}, \mathbf{b})$ is mapped to $(\mathbf{c}, D)$ with $\mathbf{a}=(2,3,2), \mathbf{b}=(2,1,2)$, $\mathbf{c}=(1,1,2)$, and $D$ is the pipe dream in the top left corner of the picture. Its transition chain is $Y(D)=((1,3),(2,2),(2,2),(1,1))$. Each vertical pair in the picture is also demonstrating the bijection for a different permutation; note the permutations on the wires agree on the vertical pairs.

A word $c$ with this property is sometimes called a sub-staircase word. The left side is the number of pairs $(\mathbf{a}, \mathbf{b})$ where $\mathbf{a} \in R(\pi)$ and $\mathbf{b}$ is a word satisfying $1 \leqslant b_{i} \leqslant a_{i}$ for each $i=1, \ldots, p$. Our bijection is between pairs $(\mathbf{a}, \mathbf{b})$ and $(\mathbf{c}, D)$ that satisfy these conditions. The bijection and its inverse are presented in the form of explicit algorithms. Moreover, both maps are uniform over the permutation $\pi$ in the sense that they have natural descriptions that explicitly involve only $(\mathbf{a}, \mathbf{b})$ (respectively, $(\mathbf{c}, D)$ ), and not $\pi$ (although of course $\pi$ can be recovered from a or $D$ ). Indeed, if we interpret permutations $\pi \in S_{n}$ as permutations of $\mathbb{Z}$ that fix all but finitely many elements, then our maps do not even explicitly involve $n$.

The outline of the bijection is quite simple given some well-known properties of Schubert polynomials, together with the bumping algorithm for reduced words. The bumping algorithm is an important tool for studying reduced words, originally introduced and developed by Little [31] and further studied by Garsia in [13]. These properties and objects will be defined in Section 2.

In the first step, we give a modification of Little's bumping algorithm that also acts on pipe dreams, and use it to give a bijective interpretation to the LascouxSchützenberger transition equation for Schubert polynomials. Essentially the same construction has been given by Buch [20, p. 11]. The key idea is to iteratively apply the corresponding transition map to $D$ until we reach the empty pipe dream, while recording a sequence of instructions that encode which inversions/insertions are needed in order to reverse the process. We call the resulting sequence a transition chain, denoted $Y(D)$.

Next we apply the bumping algorithm on reduced words and their wiring diagrams, using the reverse of the transition chain $Y(D)$ to provide instructions. The word $\mathbf{c}$ tells us where to insert new crossings; when adding the $i$ th new crossing it should become the $c_{i}$ th entry in the word. The height of the added crossing is determined by placing its feet on the wire specified by the corresponding step of the transition 
chain. Each new crossing is immediately pushed higher in value, initiating a Little bump. The result is a reduced wiring diagram for $\pi$ corresponding to a reduced word $\mathbf{a}=\left(a_{1}, a_{2}, \ldots, a_{p}\right)$. If we keep track of how many times each column is pushed in the bumping processes, we obtain a word $\mathbf{b}=\left(b_{1}, \ldots, b_{p}\right)$ of the same length such that $a_{i} \geqslant b_{i}$ for all $i$, as required. See Figure 1 for an illustration of the algorithm. It turns out that each step is reversible.

Our bijective proof extends to a $\mathfrak{q}$-analog of (1) that was conjectured by Macdonald and subsequently proved by Fomin and Stanley. To state this formula, let $\mathfrak{q}$ be a formal variable. Define the $\mathfrak{q}$-analog of a positive integer $k$ to be $[k]=[k]_{\mathfrak{q}}:=1+$ $\mathfrak{q}+\mathfrak{q}^{2}+\cdots+\mathfrak{q}^{k-1}$. The $\mathfrak{q}$-analog of the factorial $k$ ! is defined to be $[k]^{\mathbb{0}}=[k]_{\mathfrak{q}^{0}}:=$ $[k][k-1] \cdots[1]$. (We use the blackboard bold symbol ${ }^{0}$ to distinguish it from the ordinary factorial, and the symbol $\mathfrak{q}$ for the formal variable to avoid notation conflicts.) For $\mathbf{a}=\left(a_{1}, a_{2}, \ldots, a_{p}\right) \in R(\pi)$, define the co-major index to be the sum of the ascent locations:

$$
\operatorname{comaj}(\mathbf{a}):=\sum_{\substack{1 \leqslant i<p: \\ a_{i}<a_{i+1}}} i
$$

THEOREM 1.2 (Fomin and Stanley [12]). Given a permutation $\pi \in S_{n}$ with $\ell(\pi)=p$, one has

$$
\sum_{\mathbf{a}=\left(a_{1}, a_{2}, \ldots, a_{p}\right) \in R(\pi)}\left[a_{1}\right] \cdot\left[a_{2}\right] \cdots\left[a_{p}\right] \mathfrak{q}^{\operatorname{comaj}(\mathbf{a})}=[p] \stackrel{\mathbb{0}}{\mathfrak{S}_{\pi}}\left(1, \mathfrak{q}, \mathfrak{q}^{2}, \ldots, \mathfrak{q}^{n-1}\right)
$$

Continuing with the example $\pi=[3,2,1]$, we observe that the $\mathfrak{q}$-analog formula indeed holds: [1] $[2] \cdot[1] \mathfrak{q}+[2] \cdot[1] \cdot[2] \mathfrak{q}^{2}=(1+\mathfrak{q}) \mathfrak{q}+(1+\mathfrak{q})^{2} \mathfrak{q}^{2}=\left(1+\mathfrak{q}+\mathfrak{q}^{2}\right)(1+\mathfrak{q}) \mathfrak{q}=$ $[3] \stackrel{\mathfrak{g}}{ } \cdot \mathfrak{S}_{[3,2,1]}\left(1, \mathfrak{q}, \mathfrak{q}^{2}\right)$.

In 1997, Fomin and Kirillov published a further extension to Theorem 1.2. They interpreted the right side of the formula in terms of reverse plane partitions, and asked for a bijective proof. See Theorem 7.2. Using our methods together with results of Lenart [30], and Serrano and Stump [41, 42], we provide a bijective proof.

We want to comment briefly on how the bijections in this paper were found. We were fully aware of Little's bumping algorithm so we hoped it would play a role. Many details of the exact formulation we describe here were found through extensive experimentation by hand and by computer. Experimentally, we found the transition chains to be the key link between a bounded pair and its image under $M$. As the proof was written up, we chose to suppress the dependence on the transition chains in favor of clearer descriptions of the maps.

The outline of the paper is as follows. In Section 2, we give the notation and background information on reduced words, Schubert polynomials, Little bumps, etc. The key tool for our bijection comes from the Transition Equation for Schubert polynomials. We give a bijective proof of this equation in Section 3. In Section 4, we extend the Transition Equation to bounded pairs, by which we mean pairs $(\mathbf{a}, \mathbf{b})$ satisfying $1 \leqslant b_{i} \leqslant a_{i}$, as discussed above. In Section 5 , we spell out the main bijection proving Theorem 1.1. The principal specialization of Macdonald's formula given in Theorem 1.2 is described in Section 6, along with some interesting properties of the co-major index on reduced words. In Section 7, we discuss the Fomin-Kirillov theorems and how our bijection is related to them. Finally, in Section 8 we discuss some intriguing open problems and other formulas related to Macdonald's formula. 


\section{BACKGROUND}

2.1. Permutations. We recall some basic notation and definitions relating to permutations which are standard in the theory of Schubert polynomials. We refer the reader to $[27,33,34]$ for more information.

Let $S_{n}$ be the symmetric group of all permutations $\pi=[\pi(1), \ldots, \pi(n)]$ of $\{1, \ldots, n\}$. An inversion of $\pi \in S_{n}$ is an ordered pair $(i, j)$, such that $i<j$ and $\pi(i)>\pi(j)$. The length $\ell(\pi)$ is the number of inversions of $\pi$. We write $t_{i j}$ for the transposition which swaps $i$ and $j$, and we write $s_{i}=t_{i, i+1}(1 \leqslant i \leqslant n-1)$. The $s_{i}$ are called simple transpositions; they generate $S_{n}$ as a Coxeter group. Composition of permutations is defined via $\pi \tau(i):=\pi(\tau(i))$.

An alternate notation for a permutation $\pi \in S_{n}$ is its Lehmer code, or simply its code, which is the $n$-tuple

$$
\left(L(\pi)_{1}, L(\pi)_{2}, \ldots, L(\pi)_{n}\right)
$$

where $L(\pi)_{i}$ denotes the number of inversions $(i, j)$ with first coordinate $i$. Note, $0 \leqslant L(\pi)_{i} \leqslant n-i$ for all $1 \leqslant i \leqslant n$. The permutation $\pi$ is said to be dominant if its code is a weakly decreasing sequence.

2.2. REDUCED WORDS. A word is a $k$-tuple of integers. The ascent set of a word $\mathbf{a}=\left(a_{1}, \ldots, a_{k}\right)$ is $\left\{i: a_{i}<a_{i+1}\right\} \subseteq\{1, \ldots, k-1\}$. The descent set of $\mathbf{a}$ is the complement.

Let $\pi \in S_{n}$ be a permutation. A word for $\pi$ is a word $\mathbf{a}=\left(a_{1}, \ldots, a_{k}\right)$ such that $1 \leqslant a_{i}<n$ and

$$
s_{a_{1}} s_{a_{2}} \ldots s_{a_{k}}=\pi .
$$

If $k=\ell(\pi)$, then we say that a is a reduced word for $\pi$. The reduced words are precisely the minimum-length ways of representing $\pi$ in terms of the simple transpositions. For instance, the permutation $[3,2,1] \in S_{3}$ has two reduced words: $(1,2,1)$ and $(2,1,2)$. The empty word () is the unique reduced word for the identity permutation $[1,2, \ldots, n] \in$ $S_{n}$.

Write $R(\pi)$ for the set of all reduced words of the permutation $\pi$. The set $R(\pi)$ has been extensively studied, in part due to interest in Bott-Samelson varieties and Schubert calculus. Its size has an interpretation in terms of counting standard tableaux and the Stanley symmetric functions $[27,31,44]$.

Define the wiring diagram for a word $\mathbf{a}=\left(a_{1}, \ldots, a_{k}\right)$ as follows. First, for $0 \leqslant t \leqslant$ $k$, define the permutation $\pi_{t} \in S_{n}$ at time $t$ by

$$
\pi_{t}=s_{a_{1}} s_{a_{1}} \cdots s_{a_{t}} .
$$

So $\pi_{0}$ is the identity, while $\pi_{k}=\pi$. The $i$-wire of $\mathbf{a}$ is defined to be the piecewise linear path joining the points $\left(\pi_{t}^{-1}(i), t\right)$ for $0 \leqslant t \leqslant k$. We will consistently use "matrix coordinates" to describe wiring diagrams, so that $(i, j)$ refers to row $i$ (numbered from the top of the diagram) and column $j$ (numbered from the left). The wiring diagram is the union of these $n$ wires. See Figure 2 for an example.

For all $t \geqslant 1$, observe that between columns $t-1$ and $t$ in the wiring diagram for a, precisely two wires $i$ and $j$ intersect. This intersection is called a crossing. One can identify a crossing by its column $t$. We call $a_{t}$ the row of the crossing at column $t$. When the word $\mathbf{a}$ is reduced, the minimality of the length of a ensures that any two wires cross at most once. In this case, we can also identify a crossing by the unordered pair of wire labels that are involved, i.e. the pair $\left\{\pi_{t}\left(a_{t}\right), \pi_{t}\left(a_{t+1}\right)\right\}$.

Note that the terms row and column have slightly different meaning when we refer to a crossing versus a wire. The upper left corner of a wiring diagram is at $(1,0)$. When we say a crossing in row $i$ column $j$ it means the intersection of the crossing is 

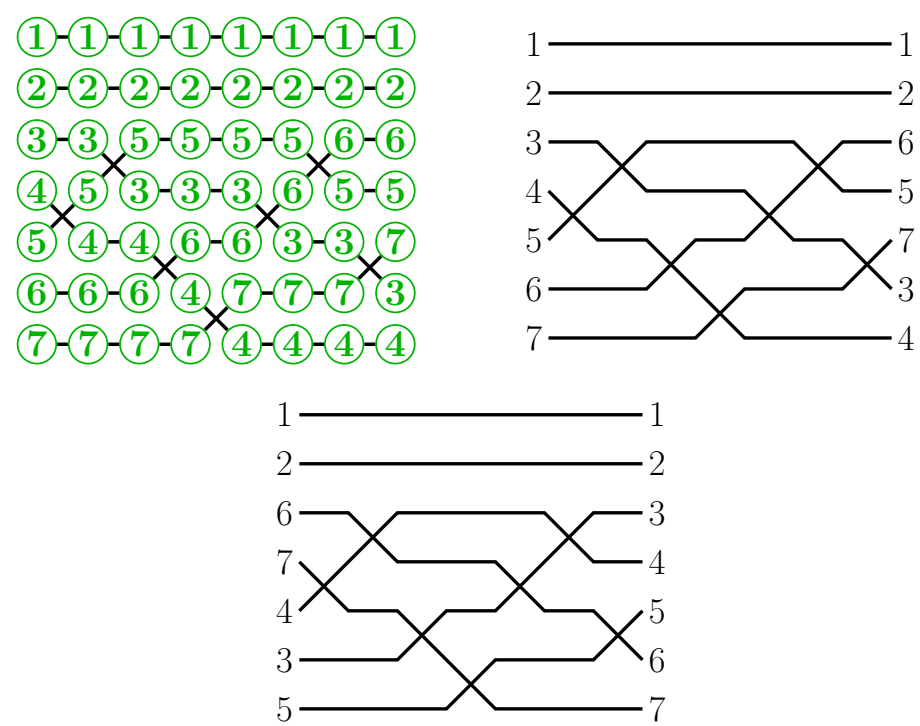

Figure 2. The wiring diagram for the reduced word $(4,3,5,6,4,3,5) \in R([1,2,6,5,7,3,4])$ annotated in three different ways: with the intermediate permutations $\pi_{t}$, the left-labeling, and the right-labeling. The crossings in columns 2 and 6 are both at row 3 .

at $\left(i+\frac{1}{2}, j-\frac{1}{2}\right)$. When we say wire $r$ is in row $i$ at column $j$, we mean that $\pi_{j}(i)=r$, so that the $r$-wire passes through the point $(i, j)$.

Observe that for $i<j$, wires $\pi(i)$ and $\pi(j)$ cross in the wiring diagram for $\mathbf{a} \in R(\pi)$ if and only if $\pi(i)>\pi(j)$. This occurs if and only if $(i, j)$ is an inversion of $\pi$, which in turn is equivalent to the wire labels $(\pi(j), \pi(i))$ being an inversion of $\pi^{-1}$. Many of the arguments below depend on the positions of the inversions for $\pi$ not for $\pi^{-1}$. Reversing any word for $\pi$ gives a word for $\pi^{-1}$. Thus, if we label the wires $1,2,3, \ldots$ in increasing order down the right side of a wiring diagram instead of the left, then the corresponding wires travel right to left, and appear in the order $\pi^{-1}$ down the left side. Thus, the $i$-wire and the $j$-wire cross in the right-labeled wiring diagram for $\mathbf{a} \in R(w)$ if and only if $(i, j)$ is an inversion of $\pi$.

The wiring diagrams shown on the first row of Figure 1 are all right-labeled wiring diagrams. For example, the word $(1,3,2)$ corresponding to the second wiring diagram from the left is a reduced word for the permutation $[2,4,1,3]=[3,1,4,2]^{-1}$.

2.3. Bounded Bumping ALGORITHM. Little's bumping algorithm [31], also known as a "Little bump", is a map on reduced words. It was introduced to study the decomposition of Stanley symmetric functions into Schur functions in a bijective way. Later, the Little algorithm was found to be related to the Robinson-Schensted-Knuth map [32] and the Edelman-Greene map [18]; it has been extended to signed permutations [2], affine permutations [25], and the subset of involutions in $S_{n}$ [17]. The key building block of our bijective proofs is an enhancement of Little's algorithm which we call the bounded bumping algorithm. We describe it below, after setting up notation. 
Definition 2.1. Let $\mathbf{a}=\left(a_{1}, \ldots, a_{k}\right)$ be a word. Define the decrement-push, increment-push, deletion and insertion of a at column $t$, respectively, to be

$$
\begin{aligned}
\mathscr{P}_{t}^{-} \mathbf{a} & =\left(a_{1}, \ldots, a_{t-1}, a_{t}-1, a_{t+1}, \ldots, a_{k}\right) ; \\
\mathscr{P}_{t}^{+} \mathbf{a} & =\left(a_{1}, \ldots, a_{t-1}, a_{t}+1, a_{t+1}, \ldots, a_{k}\right) ; \\
\mathscr{D}_{t} \mathbf{a} & =\left(a_{1}, \ldots, a_{t-1}, a_{t+1}, \ldots, a_{k}\right) ; \\
\mathscr{I}_{t}^{x} \mathbf{a} & =\left(a_{1}, \ldots, a_{t-1}, x, a_{t}, \ldots, a_{k}\right) .
\end{aligned}
$$

In [49], the notation $\mathscr{P}^{\uparrow}$ was used to represent $\mathscr{P}^{-}$, and $\mathscr{P}^{\downarrow}$ was used to represent $\mathscr{P}^{+}$based on the direction of a crossing in the wiring diagram.

Definition 2.2. Let $\mathbf{a}$ be a word. If $\mathscr{D}_{t} \mathbf{a}$ is reduced, then we say that $\mathbf{a}$ is nearly reduced at $t$.

The term "nearly reduced" was coined by Lam [24, Chapter 3], who uses " $t$-marked nearly reduced". Words that are nearly reduced at $t$ may or may not also be reduced; however, every reduced word $\mathbf{a}$ is nearly reduced at some index $t$. For instance, a reduced word a of length $k$ is nearly reduced at 1 and at $k$.

In order to define the bounded bumping algorithm, we need the following lemma, which to our knowledge first appeared in [31, Lemma 4], and was later generalized to arbitrary Coxeter systems by Lam and Shimozono using the strong exchange property. The statement can also be checked for permutations by considering the wiring diagram.

LEMma 2.3 ([25, Lemma 21]). If a is not reduced, but is nearly reduced at $t$, then $\mathbf{a}$ is nearly reduced at exactly one other column $t^{\prime} \neq t$. In the wiring diagram of $\mathbf{a}$, the two wires crossing in column $t$ cross in exactly one other column $t^{\prime}$.

DEFINITION 2.4. In the situation of Lemma 2.3, we say that $t^{\prime}$ forms a defect with $t$ in $\mathbf{a}$, and write $\operatorname{Defect}_{t}(\mathbf{a})=t^{\prime}$.

A crucial point is that the definitions of "reduced", "nearly reduced", and the Defect map make sense even if we are given only the word a, but not the corresponding permutation $\pi \in S_{n}$, nor even its size $n$. Indeed, we can take $n$ to be any integer greater than the largest element of $\mathbf{a}$; it is easily seen that the three notions coincide for all such $n$. An alternative, equivalent viewpoint is to interpret all our permutations as permutations of $\mathbb{Z}^{+}:=\{1,2, \ldots\}$ that fix all but finitely many elements; we can abbreviate such a permutation $\pi=[\pi(1), \pi(2), \ldots]$ to $\pi=[\pi(1), \ldots, \pi(n)]$ where $n$ is any integer such that all elements greater than $n$ are fixed. Let $S_{\infty}$ be the set of all such permutations on $\mathbb{Z}^{+}$.

Our central tool is a modification of the bumping algorithm introduced by Little in [31]. We call our modified version the bounded bumping algorithm. This algorithm will be used twice in the proof of Theorem 1.1, in two different contexts.

DeFinition 2.5. A word $\mathbf{b}$ is a bounded word for another word $\mathbf{a}$ if the words have the same length and $1 \leqslant b_{i} \leqslant a_{i}$ for all $i$. A bounded pair (for a permutation $\pi$ ) is an ordered pair $(\mathbf{a}, \mathbf{b})$ such that $\mathbf{a}$ is a reduced word (for $\pi$ ) and $\mathbf{b}$ is a bounded word for $\mathbf{a}$. Let BoundedPairs $(\pi)$ be the set of all bounded pairs for $\pi$.

For example, for the simple transposition $s_{k}$, the set is

$$
\text { BoundedPairs }\left(s_{k}\right)=\{((k),(i)): 1 \leqslant i \leqslant k\} .
$$

Algorithm 2.6 (Bounded Bumping Algorithm).

Input: $\left(\mathbf{a}, \mathbf{b}, t_{0}, \epsilon\right)$, where $\mathbf{a}$ is a word that is nearly reduced at $t_{0}$, and $\mathbf{b}$ is a bounded word for $\mathbf{a}$, and $\epsilon \in\{-,+\}=\{-1,+1\}$ is a direction. 

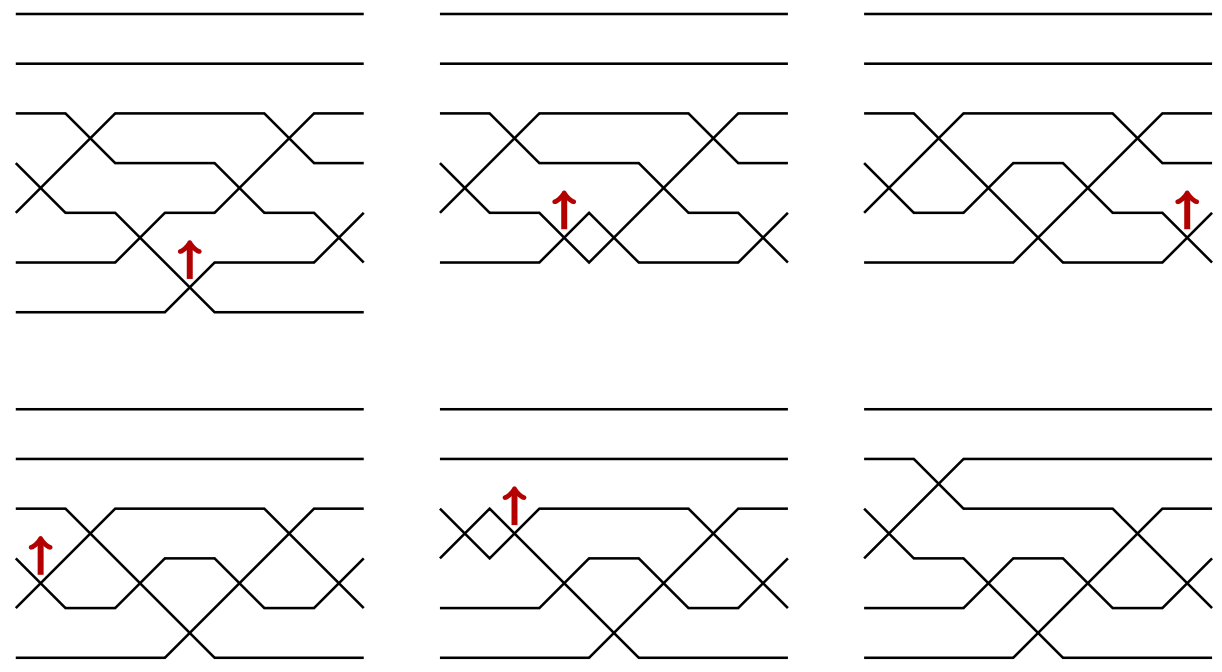

FiguRE 3. An example of the sequence of wiring diagrams for the words $\mathbf{a}^{\prime}$ which appear when running the bounded bumping algorithm on input $\mathbf{a}=(4,3,5,6,4,3,5), \mathbf{b}=(2,2,2,2,2,2,2), t_{0}=4$, and $\epsilon=$ - . The arrows indicate which crossing will move in the next step. After the first step, row 7 contains a wire with no swaps, which is therefore not shown.

Output: $\mathscr{B}_{t_{0}}^{\epsilon}(\mathbf{a}, \mathbf{b})=\left(\mathbf{a}^{\prime}, \mathbf{b}^{\prime}, i, j\right.$, outcome $)$, where $\mathbf{a}^{\prime}$ is a reduced word, $\mathbf{b}^{\prime}$ is a bounded word for $\mathbf{a}^{\prime}, i$ is the row and $j$ is the column of the last crossing pushed in the algorithm, and outcome is a binary indicator explained below.

(1) Initialize $\mathbf{a}^{\prime} \leftarrow \mathbf{a}, \mathbf{b}^{\prime} \leftarrow \mathbf{b}, t \leftarrow t_{0}$.

(2) Push in direction $\epsilon$ at column $t$, i.e. set $\mathbf{a}^{\prime} \leftarrow \mathscr{P}_{t}^{\epsilon} \mathbf{a}^{\prime}$ and $\mathbf{b}^{\prime} \leftarrow \mathscr{P}_{t}^{\epsilon} \mathbf{b}^{\prime}$.

(3) If $b_{t}^{\prime}=0$, return $\left(\mathscr{D}_{t} \mathbf{a}^{\prime}, \mathscr{D}_{t} \mathbf{b}^{\prime}, \mathbf{a}_{t}^{\prime}, t\right.$, deleted) and stop.

(4) If $\mathbf{a}^{\prime}$ is reduced, return $\left(\mathbf{a}^{\prime}, \mathbf{b}^{\prime}, \mathbf{a}_{t}^{\prime}, t\right.$, bumped) and stop.

(5) Set $t \leftarrow \operatorname{Defect}_{t}\left(\mathbf{a}^{\prime}\right)$ and return to step 2.

The principal difference between the above algorithm and Little's map $\theta_{r}$ in [31] is the presence of the bounded word $\mathbf{b}$, which indicates the number of times each column is allowed to be decremented before being deleted. The stopping rule in step 3 is not present in Little's algorithm. As discussed above, one consequence is that when $\epsilon=-$, our map can never output a word containing a 0 : if a push results in a 0 then it is immediately deleted and the algorithm stops. In contrast, in Little's original algorithm, the entire word is instead shifted by +1 in this situation, changing the permutation (and also stopping, since the word is reduced). Indeed, Little's bumping algorithm in the +1 direction on a reduced word $\mathbf{a}$ maps to $\mathbf{a}^{\prime}$ if and only if

$$
\mathscr{B}_{j}^{+}(\mathbf{a}, \mathbf{b})=\left(\mathbf{a}^{\prime}, \mathbf{b}^{\prime}, i, j, \text { bumped }\right)
$$

regardless of the choice of bounded word $\mathbf{b}$ for $\mathbf{a}$.

Since this algorithm is the main tool used in the paper, we will give several examples. In Figure 3, we show the sequence of wiring diagrams for the words $\mathbf{a}^{\prime}$ in the algorithm when it is run on the input

$$
\mathbf{a}=(4,3,5,6,4,3,5), \mathbf{b}=(2,2,2,2,2,2,2), t_{0}=4 \text {, and } \epsilon=-.
$$


The result is

$$
\mathscr{B}_{4}^{-}(\mathbf{a}, \mathbf{b})=((3,2,4,5,4,3,4),(1,1,1,1,2,2,1), 2,2, \text { bumped }) .
$$

Note that Little's bumping algorithm maps $(4,3,5,6,4,3,5)$ to $(3,2,4,5,4,3,4)$ using the exact same sequence of pushes as in Figure 3 as expected since outcome $=$ bumped.

On the other hand, with input $\widetilde{\mathbf{b}}=(2,2,2,2,2,2,1)$ the bounded bumping algorithm stops after the third push in the sequence because $\widetilde{b}_{7}=1$, so

$$
\mathscr{B}_{4}^{-}(\mathbf{a}, \widetilde{\mathbf{b}})=((4,3,4,5,4,3),(2,2,1,1,2,2), 4,7 \text {, deleted }) .
$$

Another good example for the reader to consider is when the input word $\mathbf{a}$ is a consecutive sequence such as

$$
\mathscr{B}_{1}^{-}((6,5,4,3),(3,3,3,3))=((5,4,3,2),(2,2,2,2), 2,4 \text {, bumped }) .
$$

We now make some remarks about this algorithm. The initial input word a may or may not be reduced, but, if we reach step 5 then $\mathbf{a}^{\prime}$ is always not reduced but nearly reduced at $t$, so the Defect map makes sense.

Suppose that the input word $\mathbf{a}$ is a word for a permutation $\pi \in S_{n}$. Pushes may in general result in words with elements outside the interval $[1, n-1]$. Specifically, in the case $\epsilon=+$, step 2 may result in a word $\mathbf{a}^{\prime}$ with an element $a_{t}^{\prime}=n$. As mentioned above, this can be interpreted as a word for a permutation in $S_{n+1}$. In fact, in this case the algorithm will immediately stop at step 4 , since this new word is necessarily reduced. On the other hand, in the case $\epsilon=-$, if step 2 ever results in a word with $a_{t}^{\prime}=0$, we must have $b_{t}^{\prime}=0$ as well, so the algorithm will immediately stop at step 3 , and the 0 will be deleted. Note that it is also possible for a non-zero element of $\mathbf{a}$ to be deleted at step 3 , since $b_{i}^{\prime}<a_{i}^{\prime}$ is possible. Thus, the bounded bumping algorithm clearly terminates in a finite number of steps.

The proposition below collects several technical facts about the bounded bumping algorithm that are analogous to facts proved by Little about his algorithm [31]. These statements may be checked by essentially the same arguments as in [31] - the inclusion of $\mathbf{b}$ has scant effect here.

Proposition 2.7. Let $\mathbf{a}$ be a word that is nearly reduced at $t$, let $\mathbf{b}$ be a bounded word for $\mathbf{a}$, and let $\epsilon \in\{+,-\}$. Assume $\mathscr{B}_{t}^{\epsilon}(\mathbf{a}, \mathbf{b})=\left(\mathbf{a}^{\prime}, \mathbf{b}^{\prime}, i, j\right.$, outcome $)$.

(1) Suppose a is reduced. Then, Algorithm 2.6 is reversible in the sense that we can recover the inputs by negating the direction $\epsilon$. More specifically, if outcome = deleted, then $\epsilon=-1$ and $\mathscr{B}_{j}^{-\epsilon}\left(\mathscr{I}_{j}^{i} \mathbf{a}^{\prime}, \mathscr{I}_{j}^{0} \mathbf{b}^{\prime}\right)=\left(\mathbf{a}, \mathbf{b}, \mathbf{a}_{t}, t\right.$, bumped $)$; if outcome = bumped, then $\mathscr{B}_{j}^{-\epsilon}\left(\mathbf{a}^{\prime}, \mathbf{b}^{\prime}\right)=\left(\mathbf{a}, \mathbf{b}, \mathbf{a}_{t}, t\right.$, bumped $)$.

(2) If $\mathbf{a} \in R(\pi)$, then $\mathscr{D}_{t} \mathbf{a} \in R\left(\pi t_{k, l}\right)$, where $(k<l)$ is the inversion of $\pi$ whose wires cross in column t of the right-labeled wiring diagram for $\mathbf{a}$. If outcome $=$ bumped, then $\mathbf{a}^{\prime} \in R\left(\pi t_{k, l} t_{x, y}\right)$ where $\{x<y\}$ is the crossing in column $j$ of the word $\mathbf{a}^{\prime}$ for $\pi t_{k, l} t_{x, y}$. Furthermore, if $\epsilon=+$, then $l=x$. If $\epsilon=-$, then $k=y$.

(3) Suppose $\mathscr{D}_{j} \mathbf{a} \in R(\nu)$. After every iteration of step 2 in the bounded bumping algorithm computing $\mathscr{B}_{t}^{\epsilon}(\mathbf{a}, \mathbf{b})$, the pair $\left(\mathscr{D}_{t} \mathbf{a}^{\prime}, \mathscr{D}_{t} \mathbf{b}^{\prime}\right)$ is a bounded pair for $\nu$. In particular, if outcome = deleted, then $\mathbf{a}^{\prime} \in R(\nu)$.

(4) If outcome = bumped, then the input and output words $\mathbf{a}$ and $\mathbf{a}^{\prime}$ have the same ascent set. If outcome $=$ deleted, then the ascent set of $\mathscr{I}_{j}^{i}\left(\mathbf{a}^{\prime}\right)$ is the same as the ascent set of $\mathbf{a}$.

Note that in items (3) and (4) above, the word $\mathbf{a}$ is not necessarily reduced. 

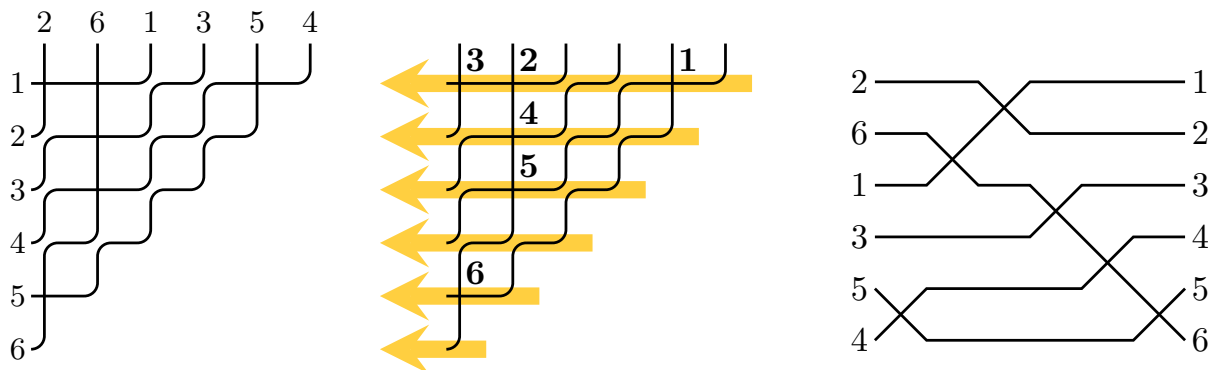

Figure 4. Left: a reduced pipe dream $D$ for $\pi=[3,1,4,6,5,2]=$ $[2,6,1,3,5,4]^{-1}$. The weight is $x^{D}=x_{1}^{3} x_{2} x_{3} x_{5}$. Middle: the reading order for the crossings, with numbers indicating position in the order. The resulting sequences of row numbers and column numbers are $\mathbf{i}_{D}=(1,1,1,2,3,5)$ and $\mathbf{j}_{D}=(5,2,1,2,2,1)$ respectively. Right: the right-labeled wiring diagram of the associated reduced word $\mathbf{r}_{D}=$ $(5,2,1,3,4,5) \in R(\pi)$.

2.4. Pipe Dreams and Schubert Polynomials. Schubert polynomials $\mathfrak{S}_{\pi}$ for $\pi \in$ $S_{n}$ are a generalization of Schur polynomials invented by Lascoux and Schützenberger in the early 1980s [27]. They have been widely used and studied over the past 30 years. An excellent summary of the early work on these polynomials appears in Macdonald's notes [33]; see Manivel's book [34] for a more recent treatment.

A pipe dream $D$ is a finite subset of $\mathbb{Z}_{+} \times \mathbb{Z}_{+}$. We will usually draw a pipe dream as a modified wiring diagram as follows. Place a + symbol at every point $(i, j) \in D$; place a pair of elbows $r$ at every other point $(i, j) \in\left(\mathbb{Z}_{+} \times \mathbb{Z}_{+}\right) \backslash D$, where again we use matrix-style coordinates. This creates wires connecting points on the left side of the diagram to points on the top. If the wires are numbered $1,2,3, \ldots$ down the left side, then the corresponding wires reading along the top of the diagram from left to right form a permutation $\pi$ of the positive integers that fixes all but finitely many values. We call $\pi^{-1}$ the permutation of $D$ following the literature.

We call the elements of a pipe dream $D \subset \mathbb{Z}_{+} \times \mathbb{Z}_{+}$crossings or occupied positions, and the elements $(i, j)$ of $\mathbb{Z}_{+} \times \mathbb{Z}_{+} \backslash D$ unoccupied positions. Each crossing involves two wires, which are said to enter the crossing horizontally and vertically.

Following the terminology for reduced words, we say that $D$ is reduced if $\pi$ is the permutation of $D$ and $\ell(\pi)=|D|$. We write $\mathcal{R} \mathcal{P}(\pi)$ for the set of all reduced pipe dreams for $\pi$. Two wires labeled $i<j$ cross somewhere in $D \in \mathcal{R P}(\pi)$ if and only if $(i, j)$ is an inversion of $\pi$. Observe that the smaller labeled wire necessarily enters the crossing horizontally in a reduced pipe dream.

As mentioned earlier, we can identify the permutation of a pipe dream with one in $S_{n}$, where all elements greater than $n$ are fixed. We only need to draw a finite number of wires in a triangular array to represent a pipe dream since for all large enough wires there are no crossings. See Figure 4 for an example.

The weight of a pipe dream $D$ is given by the product over row numbers of the crossings

$$
x^{D}:=\prod_{(i, j) \in D} x_{i}
$$

where $x_{1}, x_{2}, \ldots$ are formal variables. The Schubert polynomial can be defined as a generating function for weighted reduced pipe dreams as follows. 
Definition 2.8. The Schubert polynomial of $\pi \in S_{n}$ is defined to be

$$
\mathfrak{S}_{\pi}=\mathfrak{S}_{\pi}\left(x_{1}, x_{2}, \ldots, x_{n}\right):=\sum_{D \in \mathcal{R} \mathcal{P}(\pi)} x^{D} .
$$

For example, the second row of Figure 1 shows pipe dreams for 5 different permutations. The pipe dream in the middle of the figure is the unique reduced pipe dream for $[2,3,1,4]=[3,1,2,4]^{-1}$ so $\mathfrak{S}_{[2,3,1,4]}=x_{1} x_{2}$. The pipe dream on the left for $[1,4,3,2]$ is not the only one. There are 5 pipe dreams for $w=[1,4,3,2]$ in total and

$$
\mathfrak{S}_{[1,4,3,2]}=x_{1}^{2} x_{2}+x_{1}^{2} x_{3}+x_{1} x_{2}^{2}+x_{1} x_{2} x_{3}+x_{2}^{2} x_{3} .
$$

There are many other equivalent definitions of Schubert polynomials $[1,3,10,12$, $27,48]$. Note that pipe dreams are also called pseudo-line arrangements and $R C$ graphs in the literature. See $[21,22]$ for other geometric and algebraic interpretations of individual pipe dreams.

The following theorem is an important tool for calculating Schubert polynomials. It is a recurrence based on the lexicographically (lex) largest inversion $(r, s)$ for $\pi$ assuming $\pi \neq \mathrm{id}$, where as usual an inversion means $r<s$ and $\pi(r)>\pi(s)$. Note that $r$ is the position of the largest descent in $\pi$, and $s$ is the largest value such that $\pi(r)>\pi(s)$. If $\mathbf{a}$ is a reduced word for $\pi$, then there exists a unique column $t_{0}$ containing the $\{r, s\}$-wire crossing in the right-labeled wiring diagram for a. One can easily verify that $\ell\left(\pi t_{r s}\right)=\ell(\pi)-1$, and hence $\mathbf{a}$ is nearly reduced in column $t_{0}$. The original proof due to Lascoux and Schützenberger [29] uses Monk's formula for computing products of Schubert classes in the cohomology ring of the flag manifold. See also [33, 4.16]. We give a bijective proof using pipe dreams in the next section.

TheOREM 2.9 (Transition Equation for Schubert polynomials [29]). For all permutations $\pi$ with $\pi \neq \mathrm{id}$, the Schubert polynomial $\mathfrak{S}_{\pi}$ is determined by the recurrence

$$
\mathfrak{S}_{\pi}=x_{r} \mathfrak{S}_{\nu}+\sum_{\substack{q<r: \\ \ell(\pi)=\ell\left(\nu t_{q r}\right)}} \mathfrak{S}_{\nu t_{q r}}
$$

where $(r, s)$ is the lex largest inversion in $\pi$, and $\nu=\pi t_{r s}$. The base case of the recurrence is $\mathfrak{S}_{\mathrm{id}}=1$.

Continuing the example above, the lex largest inversion for $w=[1,4,3,2]$ is $(3,4)$ so

$$
\mathfrak{S}_{[1,4,3,2]}=x_{3} \mathfrak{S}_{[1,4,2,3]}+\mathfrak{S}_{[2,4,1,3]} .
$$

The lex largest inversion for $[2,4,1,3]$ is $(2,4)$ so

$$
\mathfrak{S}_{[2,4,1,3]}=x_{2} \mathfrak{S}_{[2,3,1,4]}+\mathfrak{S}_{[3,2,1,4]} .
$$

If we continue to use the Transition Equation, we find $\mathfrak{S}_{[3,2,1,4]}=x_{1}^{2} x_{2}, \mathfrak{S}_{[2,3,1,4]}=$ $x_{1} x_{2}$ and $\mathfrak{S}_{[1,4,2,3]}=x_{2} \mathfrak{S}_{[1,3,2,4]}+\mathfrak{S}_{[3,1,2,4]}=x_{2}\left(x_{1}+x_{2}\right)+x_{1}^{2}$. Therefore, we can rederive (3) via the Transition Equation as well.

DeFinition 2.10. We define the inversion order $\prec$ on permutations as follows. Given $\pi \in S_{\infty}$, let $\operatorname{Inv}(\pi)$ be the ordered list of inversions in reverse lex order. Note, $\operatorname{Inv}(\pi)$ begins with the lex largest inversion of $u$. Then, for $\tau, \pi \in S_{\infty}$, we say $\tau \prec \pi$ provided $\operatorname{Inv}(\tau)<\operatorname{Inv}(\pi)$ in lex order as lists. For example, $\operatorname{Inv}([1432])=((3,4),(2,4),(2,3))$ and $\operatorname{Inv}([2413])=((2,4),(2,3),(1,3))$, so $[2413] \prec[1432]$.

REMARK 2.11. All of the permutations on the right hand side of (4) are strictly smaller than $\pi$ in inversion order by construction. Furthermore, the permutations on the right hand side of (4) are in $S_{n}$ provided $\pi \in S_{n}$. Hence there are only a finite number of terms in the expansion of a Schubert polynomial. We will apply induction over this finite set in the bijective proofs that follow. 


\section{Bijective proof of the Transition Equation}

In this section, we give a bijective proof of the Transition Equation for Schubert polynomials, Theorem 2.9. The Transition Algorithm described here is a key tool for proving Theorem 1.1. We begin by describing how the bounded bumping algorithm acts on reduced pipe dreams.

A pipe dream for a permutation $\pi$ may be interpreted as a bounded pair of a special type for the same $\pi$. To make this more precise, order the crossings in $D$ in the order given by reading rows from top to bottom, and from right to left within each row. We call this the reading order on $D$. We construct three words from the ordered list of crossings: the row numbers of the crossings $\mathbf{i}_{D}=\left(i_{1}, i_{2}, \ldots, i_{p}\right)$, the column numbers $\mathbf{j}_{D}=\left(j_{1}, j_{2}, \ldots, j_{p}\right)$ and the diagonal numbers $\mathbf{r}_{D}=\left(i_{1}+j_{1}-1, i_{2}+j_{2}-1, \ldots, i_{p}+\right.$ $\left.j_{p}-1\right)=\mathbf{j}_{D}+\mathbf{i}_{D}-\mathbf{1}$. Any two of $\mathbf{i}_{D}, \mathbf{j}_{D}, \mathbf{r}_{D}$ suffice to determine $D$. In this paper, we will encode $D$ by the biword $\left(\mathbf{r}_{D}, \mathbf{j}_{D}\right)$ departing from the literature which typically uses $\left(\mathbf{r}_{D}, \mathbf{i}_{D}\right)$.

If $D$ is a pipe dream for $\pi$ then it is easy to see that $\mathbf{r}_{D}$ is a word for $\pi$. And $D$ is reduced if and only if $\mathbf{r}_{D}$ is. Furthermore, the column numbers $\mathbf{j}_{D}$ always form a bounded word for $\mathbf{r}_{D}$. Although the bounded pair $\left(\mathbf{r}_{D}, \mathbf{j}_{D}\right)$ determines $D$, not every bounded pair corresponds to a pipe dream. In fact, a bounded pair $(\mathbf{a}, \mathbf{b})=\left(\left(a_{1}, \ldots, a_{p}\right),\left(b_{1}, \ldots, b_{p}\right)\right)$ corresponds to a pipe dream if and only if the list $\left[\left(i_{1}, b_{1}\right), \ldots,\left(i_{p}, b_{p}\right)\right]$ has $p$ distinct elements listed in the reading order, where $i_{k}=a_{k}-b_{k}+1$. Equivalently, $(\mathbf{a}, \mathbf{b})$ corresponds to a pipe dream if and only if the pairs $\left(i_{1},-b_{1}\right), \ldots,\left(i_{p},-b_{p}\right)$ are in strictly increasing lex order.

For example, Figure 4 shows the pipe dream $D$ corresponding with reduced word $\mathbf{r}_{D}=(5,2,1,3,4,5)$, row numbers $\mathbf{i}_{D}=(1,1,1,2,3,5)$, and column numbers $\mathbf{j}_{D}=$ $(5,2,1,2,2,1)$.

Using the biword $\left(\mathbf{r}_{D}, \mathbf{j}_{D}\right)$ to encode a reduced pipe dream $D$, we can apply the bounded bumping algorithm to $D$ in either direction and for any $t_{0}$ where $\mathbf{r}_{D}$ is nearly reduced. One can observe that the bounded pairs encountered during the steps of the bounded bumping algorithm do not all encode pipe dreams, but it will turn out that the departures from "pipe dream encoding status" are temporary, and have a straightforward structure that will be analyzed in the proof of Lemma 3.1 below.

LEMma 3.1. Let $D$ be a reduced pipe dream and suppose that $r_{D}$ is nearly reduced at t. Let $\epsilon \in\{+,-\}$ and write

$$
\mathscr{B}_{t}^{\epsilon}\left(\mathbf{r}_{D}, \mathbf{j}_{D}\right)=\left(\mathbf{a}^{\prime}, \mathbf{b}^{\prime}, i, j, \text { outcome }\right) .
$$

Then the bounded pair $\left(\mathbf{a}^{\prime}, \mathbf{b}^{\prime}\right)$ also encodes a reduced pipe dream.

Proof. Consider the effect of the bounded bumping algorithm in terms of pipe dreams. To be concrete, assume $\epsilon=-$, the case $\epsilon=+$ being similar. Observe that when we initially decrement-push $\left(\mathbf{r}_{D}, \mathbf{j}_{D}\right)$ in column $t$, it has the effect of moving the $t^{t h}$ crossing in the reading order on $D$, say in position $(i, j) \in D$, one column to the left to position $(i, j-1)$. If this location is already occupied, $(i, j-1) \in D$, then $\mathscr{P}_{t}^{-} r_{D}$ returns a nearly reduced word with identical letters in positions $t$ and $t+1$. The resulting bounded pair does not encode a pipe dream. Then, the next step of the bounded bumping algorithm will decrement-push at $t+1$. If $(i, j-2) \in D$ also, then $\mathbf{a}^{\prime}=\mathscr{P}_{t+1}^{-} \mathscr{P}_{t}^{-} r_{D}$ will again have duplicate copies of the letter $i+j-1$ in positions $t+1$ and $t+2$ so the next decrement-push will be in position $t+2$, and so on. Note that since the algorithm decrement-pushes both of the words in the bounded pair in the same position at each iteration, the entrywise differences $\mathbf{a}^{\prime}-\mathbf{b}^{\prime}=\mathbf{r}_{D}-\mathbf{j}_{D}$ agree, so the original row numbers $\mathbf{i}_{D}$ are maintained unless a deletion occurs. 
We can group the push steps along one row so a decrement-push in position $(i, j)$ pushes all of the adjacent +'s to its left over by one as a stack. Thus, the effect of the bounded bumping algorithm on the pipe dream amounts to a sequence of such "stack pushes". If at the end of a stack push, a + in column 1 of the pipe dream is decrement-pushed, the bounded bump algorithm terminates by deleting that position because there will be a 0 in the bounded word. Otherwise, a stack push ends with a bounded pair that corresponds to a pipe dream, which may or may not be reduced. If it is reduced, the algorithm stops and returns outcome = bumped. Otherwise, we find the defect and continue with another stack push in a different row. In either case, the final bounded pair $\left(\mathbf{a}^{\prime}, \mathbf{b}^{\prime}\right)$ encodes a reduced pipe dream.

Next we give the promised bijective proof of the Transition Equation for Schubert polynomials using pipe dreams. The bijection we give was independently observed by Anders Buch [20, p. 11]. The proof will involve several technical steps, Lemmas 3.6 to 3.9 , which are stated and proved after the main argument.

Proof of Theorem 2.9. In the case $\pi=\mathrm{id}$, we have $\mathfrak{S}_{\pi}=1$ so the theorem holds trivially. Assume $\pi \neq \mathrm{id}$. Recall $\nu=\pi t_{r, s}$, and let

$$
\mathcal{U}(\pi):=\mathcal{R} \mathcal{P}(\nu) \cup \bigcup_{\substack{q<r: \\ \ell(\pi)=\ell\left(\nu t_{q r}\right)}} \mathcal{R P}\left(\nu t_{q r}\right) .
$$

We think of $\nu=\nu t_{r, r}$ so each pipe dream in $\mathcal{U}(\pi)$ is for a permutation of the form $\nu t_{q, r}$ with $1 \leqslant q \leqslant r$, though not all such $\nu t_{q, r}$ necessarily occur.

By definition, the left side of (4) is the sum of $x^{D}$ over all $D \in \mathcal{R} \mathcal{P}(\pi)$. Similarly, the right side can be expressed as a sum over all reduced pipe dreams $E \in \mathcal{U}(\pi)$. Each such $E$ contributes either $x_{r} x^{E}$ or $x^{E}$ respectively to the sum on the right side. We will give a bijection $T_{\pi}: \mathcal{R P}(\pi) \longrightarrow \mathcal{U}(\pi)$ that preserves weight, except in the cases $T_{\pi}(D)=E \in \mathcal{R} \mathcal{P}(\nu)$, where the weight will change by $x_{r}$, so $x^{D}=x_{r} x^{E}$.

Algorithm 3.2 (Transition Map). Suppose $\pi \neq \mathrm{id}$ is given, and let $(r, s)$ and $\nu$ be defined as in Theorem 2.9 .

Input: $D$, a non-empty reduced pipe dream for $\pi$ encoded as the biword $\left(\mathbf{r}_{D}, \mathbf{j}_{D}\right)$. Output: $T_{\pi}(D)=E \in \mathcal{U}(\pi)$.

(1) Let $t_{0}$ be the unique column containing the $\{r, s\}$-wiring crossing in the rightlabeled wiring diagram for $\mathbf{r}_{D}$.

(2) Compute $\mathscr{B}_{t_{0}}^{-}\left(\mathbf{r}_{D}, \mathbf{j}_{D}\right)=\left(\mathbf{a}^{\prime}, \mathbf{b}^{\prime}, i, j\right.$, outcome $)$.

(3) If outcome $=$ deleted, then we will show in Lemma 3.6 that $i=r-1, j=\ell(\pi)$, and $\left(\mathbf{a}^{\prime}, \mathbf{b}^{\prime}\right)$ encodes a pipe dream $E \in \mathcal{R} \mathcal{P}(\nu) \subset \mathcal{U}(\pi)$. Return $E$ and stop.

(4) If outcome $=$ bumped, then we will show in Lemma 3.7 that $\left(\mathbf{a}^{\prime}, \mathbf{b}^{\prime}\right)$ encodes a pipe dream $E \in \mathcal{R} \mathcal{P}\left(\nu t_{q r}\right)$ for some $q<r$ with $\ell(\pi)=\ell\left(\nu t_{q r}\right)$. Thus, $E \in \mathcal{U}(\pi)$. Return $E$ and stop.

See Example 3.4 below. The inverse map $T_{\pi}^{-1}(E)$ again has two cases.

Algorithm 3.3 (Inverse Transition Map). Suppose $\pi \neq \mathrm{id}$ is given, and let $(r, s)$ and $\nu$ be defined as in Theorem 2.9 .

Input: $E \in \mathcal{U}(\pi)$ a reduced pipe dream encoded by the biword $\left(\mathbf{r}_{E}, \mathbf{j}_{E}\right)$. In particular $E \in \mathcal{R} \mathcal{P}\left(\nu t_{q, r}\right)$ for some $1 \leqslant q \leqslant r$.

Output: $T_{\pi}^{-1}(E)=D$, a reduced pipe dream for $\pi$.

(1) If $q=r$, then $E \in \mathcal{R} \mathcal{P}(\nu)$. Set $j \leftarrow \ell(\pi), \mathbf{g} \leftarrow \mathscr{I}_{j}^{r-1}\left(\mathbf{r}_{E}\right), \mathbf{h} \leftarrow \mathscr{I}_{j}^{0}\left(\mathbf{j}_{E}\right)$.

(2) If $q<r$, set $\mathbf{g} \leftarrow \mathbf{r}_{E}$ and $\mathbf{h} \leftarrow \mathbf{j}_{E}$. Let $j$ be the column containing the $\{q, r\}$-crossing in the right-labeled wiring diagram of $\mathbf{r}_{E}$ which must exist since $E \in \mathcal{R} \mathcal{P}\left(\nu t_{q, r}\right) \subset \mathcal{U}(\pi)$. 
(3) Compute $\mathscr{B}_{j}^{+}(\mathbf{g}, \mathbf{h})=\left(\mathbf{g}^{\prime}, \mathbf{h}^{\prime}, i^{\prime}, t\right.$, bumped $)$. Here the outcome will always be bumped since we are applying increment-pushes. Lemma 3.9 below shows that $\left(\mathbf{g}^{\prime}, \mathbf{h}^{\prime}\right)$ encodes a pipe dream $D \in \mathcal{R} \mathcal{P}(\pi)$.

(4) Return D and stop.

We claim that $T_{\pi}$ is an injection. First note that, by Proposition $2.7(1)$, the bounded bumping algorithm is reversible given the column $j$ of the final push and, in addition, in the case the outcome is deleted, the value $i$ of the letter omitted. Thus, to prove injectivity, let $E \in \mathcal{U}(\pi)$, then $E \in \mathcal{R} \mathcal{P}\left(\nu t_{q, r}\right)$ for some $q \leqslant r$. We need to show $i$ and $j$ can be determined from $q$.

If $q=r$, then the final push in the bounded bumping algorithm was in the last position so $i=r-1$ and $j=\ell(\pi)$. If $q<r$ then $(q, r)$ is an inversion in $\pi t_{r, s} t_{q, r}$. Lemma 3.7 shows that $j$ is determined by the unique column of the right-labeled wiring diagram of $r_{E}$ containing the $\{q, r\}$-wire crossing which must exist since it corresponds to an inversion. Thus, $T_{\pi}^{-1} T_{\pi}(D)=D$ so if $T_{\pi}(D)=E=T_{\pi}\left(D^{\prime}\right)$ then $D=D^{\prime}$.

Similarly, for all $E \in \mathcal{U}(\pi)$ we have $T_{\pi} T_{\pi}^{-1}(E)=E$ so $T_{\pi}$ is surjective. Therefore, $T_{\pi}$ is a bijection.

Finally, we show that $T_{\pi}$ is weight preserving. Say $D \in \mathcal{R} \mathcal{P}(\pi)$ and $T_{\pi}(D)=$ $(E,(q, r))$. Recall that if the row numbers $\mathbf{i}_{D}=\left(i_{1}, \ldots, i_{p}\right)$, then $x^{D}=x_{i_{1}} \cdots x_{i_{p}}$ The row numbers are determined by $\mathbf{i}_{D}=\mathbf{r}_{D}-\mathbf{j}_{D}+\mathbf{1}$ so they are preserved by each push step in the bounded bumping algorithm since $r_{k}-j_{k}$ is preserved for each $k$. If $q<r$, then the algorithm terminates with $x^{D}=x^{E}$. If $q=r$ then the algorithm terminates when the $p$ th position is deleted and at that point $i_{p}=(r-1)-0+1=r$ so $x^{D}=x_{r} x^{E}$.

EXAMPLE 3.4. If $D$ is the pipe dream on the left in Figure 1, then the corresponding permutation is $\pi=[1,4,3,2]$. The lex largest inversion of $\pi$ is $(3,4)$ so $\nu=\pi t_{3,4}=$ $[1,4,2,3]$. The $\{3,4\}$-crossing is circled. Using the biword encoding of $D$, we have $\mathbf{r}_{D}=(2,3,2)$ and $\mathbf{j}_{D}=(2,2,1)$. To compute $T_{\pi}(D)$, we initiate the bump at $t_{0}=1$ since the $\{3,4\}$-crossing is first in reading order on $D$.

$$
\mathscr{B}_{1}^{-}((2,3,2),(2,2,1))=((1,3,2),(1,2,1), 1,1, \text { bumped }) .
$$

It requires just one push map since $(1,3,2)$ is reduced. The crossing in column 1 in the right-labeled wiring diagram for $(1,3,2)$ is between wires $(1,3)$. Thus, $T_{\pi}(D)=$ $E \in \mathcal{R} \mathcal{P}\left(\nu t_{1,3}\right)$ where $E$ is the pipe dream encoded by $\mathbf{r}_{E}=(1,3,2)$ and $\mathbf{j}_{E}=(1,2,1)$. Observe that $E$ is the second pipe dream in Figure 1.

EXAMPLE 3.5. In Figure 5, we give a more complicated example of computing $T_{\pi}(D)$. Note that a defect can occur either above or below the pushed crossing. Going from the fourth to the fifth pipe dream, two consecutive pushes on the same row are combined into one step. This is an example of a nontrivial "stack push".

Lemma 3.6. Assume the notation in Theorem 2.9 and the definition of the transition map $T_{\pi}$. Let $D \in \mathcal{R} \mathcal{P}(\pi)$. If

$$
\mathscr{B}_{t_{0}}^{-}\left(\mathbf{r}_{D}, \mathbf{j}_{D}\right)=\left(\mathbf{a}^{\prime}, \mathbf{b}^{\prime}, i, j, \text { deleted }\right),
$$

then $i=r-1, j=\ell(\pi)$, and $\left(\mathbf{a}^{\prime}, \mathbf{b}^{\prime}\right)$ encodes a pipe dream $E \in \mathcal{R} \mathcal{P}(\nu)$ so $T_{\pi}(D) \in$ $\mathcal{U}(\pi)$.

Proof. The fact that $\left(\mathbf{a}^{\prime}, \mathbf{b}^{\prime}\right)$ encodes a pipe dream $E \in \mathcal{R} \mathcal{P}(\nu)$ follows directly from Proposition 2.7(3), Lemma 3.1 and the construction of $\nu$ and $t_{0}$.

To show $i=r-1$ and $j=\ell(\pi)$, it suffices to prove that the last step of the bounded bumping algorithm stack pushed a crossing in position $(r, 1)$ into the 0th column and 

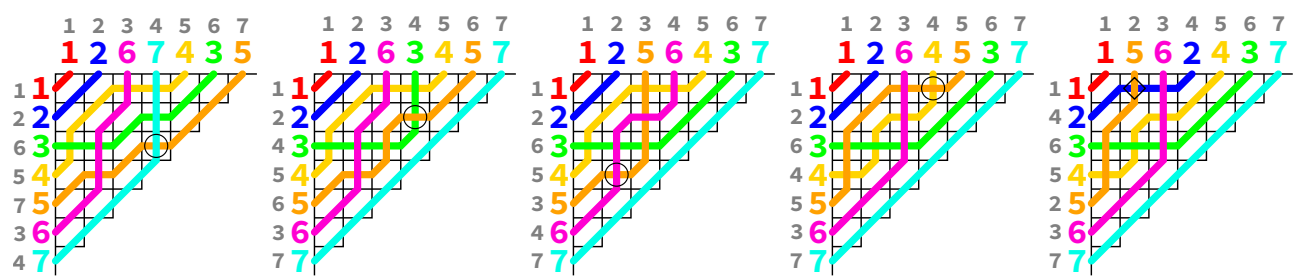

Figure 5. If $D$ is the pipe dream on the left, then $T_{\pi}(D)$ is the pipe dream on the right. In between we show the stack pushes in the bounded bumping algorithm. The crossing initiating a stack push is circled for each step, and the final crossing which moved in the last step is marked with a diamond. Here, $\pi=$ [1265734], hence $\pi^{-1}=[1267435], r=5, s=7, \nu=[1265437]$. In this case, $T_{\pi}(D)$ is a pipe dream for $\nu t_{25}=[1465237]$ so $q=2$.

out of the pipe dream. This is because $r$ is the last descent of $\pi$ so there cannot be any crossings in the reading order on $D$ after $(r, 1)$.

Say the $\{r, s\}$-wire crossing in $D$ is in row $x$ column $y$. The bumping algorithm is initiated with a stack push to the left starting at position $(x, y)$. The $r$-wire enters $(x, y)$ horizontally since $r<s$ and $D$ is a reduced pipe dream. If there is no empty position in row $x$ to the left of column $y$, then $x=r$ since the wires are labeled increasing down the left side of $D$ by definition of a pipe dream. Otherwise, if $y^{\prime}$ is the largest column such that $y^{\prime}<y$ and $\left(x, y^{\prime}\right) \notin D$, then the initial decrement-stack push on $D$ would result in the pipe dream $E=D-(x, y) \cup\left(x, y^{\prime}\right)$. Let $s^{\prime}$ be the wire crossing with the $r$-wire at $\left(x, y^{\prime}\right)$ in $E$. Now, $E$ cannot be reduced since outcome $=$ deleted in the bounded bumping algorithm. So by Lemma 2.3, we know there exists exactly one other position in $E$ where wires $r$ and $s^{\prime}$ cross, say in position $\left(x^{\prime \prime}, y^{\prime \prime}\right)$. By analyzing the possible wire configurations for two wires to cross exactly twice in a pipe dream, we see that the $r$ wire is the horizontal wire crossing in the defect position $\left(x^{\prime \prime}, y^{\prime \prime}\right)$ in $E$. Furthermore, $D-(x, y), E-\left(x, y^{\prime}\right), E-\left(x^{\prime \prime}, y^{\prime \prime}\right) \in \mathcal{R} \mathcal{P}(\nu)$.

Update $x \leftarrow x^{\prime \prime}, y \leftarrow y^{\prime \prime}$ and apply another stack push at $(x, y)$. Recursively applying the same argument we see that the only crossing in $D$ that can be pushed into column 0 must be in position $(r, 1)$.

Lemma 3.7. Assume the notation in Theorem 2.9 and the definition of the transition map $T_{\pi}$. Let $D \in \mathcal{R} \mathcal{P}(\pi)$. While computing $T_{\pi}(D)$, if

$$
\mathscr{B}_{t_{0}}^{-}\left(\mathbf{r}_{D}, \mathbf{j}_{D}\right)=\left(\mathbf{a}^{\prime}, \mathbf{b}^{\prime}, i, j, \text { bumped }\right),
$$

then the crossing in column $j$ of the right-labeled wiring diagram of $\mathbf{a}^{\prime}$ corresponds with the $r$-wire and the $q$-wire for some $q<r$ such that $\ell(\pi)=\ell\left(\nu t_{q r}\right)$. Therefore, $\left(\mathbf{a}^{\prime}, \mathbf{b}^{\prime}\right)$ encodes a pipe dream $E \in \mathcal{R} \mathcal{P}\left(\nu t_{q r}\right) \subset \mathcal{U}(\pi)$.

Proof. The proof follows from Proposition 2.7(2) and Lemma 3.1.

Given the notation established in Theorem 2.9, we can state an important consequence of the proof of Little's main theorem [31, Theorem 7]. We will use this theorem to prove Lemma 3.9. Recall that Little's bumping algorithm in the +1 direction on reduced words maps a maps to $\mathbf{a}^{\prime}$ if and only if

$$
\mathscr{B}_{j}^{+}(\mathbf{a}, \mathbf{b})=\left(\mathbf{a}^{\prime}, \mathbf{b}^{\prime}, i, t, \text { bumped }\right)
$$

for any (equivalently every) bounded word $\mathbf{b}$ for $\mathbf{a}$. 
THEOREM 3.8 ([31]). Assume the notation in Theorem 2.9. Further assume there exists some $q<r$ such that $\ell(\pi)=\ell\left(\nu t_{q r}\right)$. Given any bounded pair $(\mathbf{a}, \mathbf{b})$ for $\nu t_{q r}$, let $j$ be the column of the $\{q, r\}$-wire crossing in $\mathbf{a}$. If

$$
\mathscr{B}_{j}^{+}(\mathbf{a}, \mathbf{b})=\left(\mathbf{a}^{\prime}, \mathbf{b}^{\prime}, i, t, \text { bumped }\right),
$$

then, $\mathbf{a}^{\prime} \in R(\pi)$ and $t$ is the column containing the $\{r, s\}$-crossing in the wiring diagram of $\mathbf{a}^{\prime}$.

Lemma 3.9. Assume the notation in Theorem 2.9. Let $E \in \mathcal{R} \mathcal{P}\left(\nu t_{q, r}\right) \subset \mathcal{U}(\pi)$ for some $1 \leqslant q<r$. Assume $E$ is encoded by the bounded pair $\left(\mathbf{r}_{E}, \mathbf{j}_{E}\right), j$ is the column of the $\{q, r\}$-crossing in $\mathbf{r}_{E}$, and

$$
\mathscr{B}_{j}^{+}(\mathbf{a}, \mathbf{b})=\left(\mathbf{a}^{\prime}, \mathbf{b}^{\prime}, i, t, \text { bumped }\right) .
$$

Then $\left(\mathbf{a}^{\prime}, \mathbf{b}^{\prime}\right)$ encodes a pipe dream $D \in \mathcal{R} \mathcal{P}(\pi)$ and $t$ is the column containing the $\{r, s\}$-crossing in the wiring diagram of $\mathbf{a}^{\prime}$.

Proof. By Lemma 3.1, we know $\left(\mathbf{a}^{\prime}, \mathbf{b}^{\prime}\right)$ encodes a reduced pipe dream $D$ for some permutation with the same length as $\pi$. By Theorem 3.8, $\mathbf{a}^{\prime} \in R(\pi)$, so we conclude $D \in \mathcal{R} \mathcal{P}(\pi)$. The column of the $\{r, s\}$-crossing also follows from the theorem.

This completes all the lemmas needed for Theorem 2.9. Finally in this section, we introduce the transition chains. The transition chains will appear in the examples of the main bijection for Macdonald's identity. However, these chains do not appear in the formal proof of Theorem 1.1 explicitly.

Recall that to compute a Schubert polynomial $\mathfrak{S}_{\pi}$, one applies the Transition Equation recursively until the expansion is given as a positive sum of monomials. We could also use the Transition Map $T_{\pi}$ repeatedly to see exactly what happens to each pipe dream in $\mathcal{R} \mathcal{P}(\pi)$ in this process. The transition chain records these steps in a way that enables the process to be reversed.

Definition 3.10. Given $D \in \mathcal{R} \mathcal{P}(\pi)$, define the associated transition chain $Y(D)$ for $\pi$ recursively as follows. If $D$ is the empty pipe dream, then $D \in \mathcal{R} \mathcal{P}$ (id) and $Y(D):=$ (), the empty list. If $D \in \mathcal{R} \mathcal{P}(\pi)$ is not empty, compute $T_{\pi}(D)=E \in \mathcal{R} \mathcal{P}\left(\pi t_{r, s} t_{q, r}\right)$ and $Y(E)=\left(\left(q_{k-1}, r_{k-1}\right), \ldots,\left(i_{2}, r_{2}\right),\left(i_{1}, r_{1}\right)\right)$. Prepend $(q, r)$ onto the front, so that

$$
Y(D):=\left((q, r),\left(q_{k-1}, r_{k-1}\right), \ldots,\left(q_{2}, r_{2}\right),\left(q_{1}, r_{1}\right)\right) .
$$

For example, starting with the pipe dream $D$ encoded by $((2,3,2),(1,2,2))$ on the left in Figure 1, we apply transition maps along the second row until the we get to the empty pipe dream. This sequence of pipe dreams goes along with the following data.

\begin{tabular}{|c|c|c|c|c|}
\hline$\pi$ & $(r, s)$ & $\left(r_{D}, j_{D}\right)$ & $\left(r_{E}, j_{E}\right)$ & $(q, r)$ \\
\hline$[1,4,3,2]$ & $(3,4)$ & $(232,122)$ & $(132,122)$ & $(1,3)$ \\
{$[2,4,1,3]$} & $(2,4)$ & $(132,122)$ & $(12,11)$ & $(2,2)$ \\
{$[2,3,1,4]$} & $(2,3)$ & $(12,11)$ & $(1,1)$ & $(2,2)$ \\
{$[2,1,3,4]$} & $(1,2)$ & $(1,1)$ & $()$, & $(1,1)$ \\
\hline
\end{tabular}

Thus, $Y(D)=((1,3),(2,2),(2,2),(1,1))$.

COROLlary 3.11. The reduced pipe dreams for $\pi$ and the transition chains for $\pi$ are in bijection via the map $Y$.

Proof. This statement clearly holds for id. By induction on inversion order and Remark 2.11, we can assume $Y$ maps all pipe dreams in $\mathcal{U}(\pi)$ bijectively to their transition chains. The claim now follows since $T_{\pi}^{-1}$ is a bijection and the observation that the computation in Algorithm 3.3 only relies on the input $E \in \mathcal{U}(\pi)$ and the pair $(q, r)$. 


\section{Transition Equation for Bounded Pairs}

In the last section, the Transition Equation for Schubert polynomials was proved via a bijection on reduced pipe dreams (which can be interpreted as bounded pairs of a special kind). Next we give an analogue of the Transition Equation for the enumeration of all bounded pairs of a permutation, together with a bijective proof. Combining the two bijections will lead to our bijective proof of Theorem 1.1. Some terms are defined here in more generality than we need for this proof - they will be used later in Section 6 .

Definition 4.1. Fix $\pi \in S_{n}$ of length p. Given $\mathbf{a} \in R(\pi)$ and $\mathbf{b}$ a bounded word for $\mathbf{a}$, let $x^{\mathbf{a}}:=x_{1}^{a_{1}} x_{2}^{a_{2}} \cdots x_{p}^{a_{p}}$ and similarly for $y^{\mathbf{b}}$. Let $\mathfrak{q}$ be an formal variable, and let $\mathbf{x}=\left(x_{1}, x_{2}, \ldots, x_{p}\right)$ and $\mathbf{y}=\left(y_{1}, y_{2}, \ldots, y_{p}\right)$ be two alphabets of formal variables. Define the bounded pair polynomial to be

$$
\mathfrak{F}_{\pi}(\mathbf{x}, \mathbf{y} ; \mathfrak{q}):=\sum_{(\mathbf{a}, \mathbf{b})} x^{\mathbf{a}} y^{\mathbf{b}} \mathfrak{q}^{\operatorname{comaj}(\mathbf{a})}
$$

where the sum is over all bounded pairs $(\mathbf{a}, \mathbf{b})$ for $\pi$. For $\pi=\mathrm{id}$, set $\mathfrak{F}_{\pi}(\mathbf{x}, \mathbf{y} ; \mathfrak{q}):=1$. Thus, setting all of the variables to 1 gives the number of bounded pairs for $\pi$ :

$$
\mathfrak{F}_{\pi}(\mathbf{1})=\mathfrak{F}_{\pi}(\mathbf{1}, \mathbf{1} ; 1)=\sum_{\left(a_{1}, a_{2}, \ldots, a_{p}\right) \in R(\pi)} a_{1} \cdot a_{2} \cdots a_{p} .
$$

For example, $\mathfrak{F}_{s_{k}}(\mathbf{1})=k$ for a simple transposition $s_{k}$. Also, $\mathfrak{F}_{[3,2,1]}=6$ as mentioned in the introduction.

THEOREM 4.2 (Transition Equation for Bounded Pairs). For all permutations $\pi$ such that $\ell(\pi)=p>0$, the number of bounded pairs satisfies the following recursive formula:

$$
\mathfrak{F}_{\pi}(\mathbf{1})=p \mathfrak{F}_{\nu}(\mathbf{1})+\sum_{\substack{q<r: \\ \ell(\pi)=\ell\left(\nu t_{q r}\right)}} \mathfrak{F}_{\nu t_{q r}}(\mathbf{1}),
$$

where $(r, s)$ is the lex largest inversion of $\pi$, and $\nu=\pi t_{r s}$. The base case of the recurrence is $\mathfrak{F}_{\text {id }}(\mathbf{1})=1$.

Observe the same permutations appear in the right hand side of (7) as in (4) so Remark 2.11 applies here as well.

Proof. Similarly to the proof of Theorem 2.9, we give a bijective proof of this recurrence by defining a map $B T_{\pi}$ that maps $\operatorname{BoundedPairs}(\pi)$ to

$$
\mathcal{X}(\pi):=(\text { BoundedPairs }(\nu) \times[1, p]) \cup \bigcup_{\substack{q<r: \\ l(\pi)=l\left(\nu t_{q r}\right)}} \operatorname{BoundedPairs}\left(\nu t_{q r}\right) \times\{0\}
$$

whenever $p=\ell(\pi)>0$.

Algorithm 4.3 (Bounded Transition). Suppose $\pi \neq \mathrm{id}$ is given, and let $(r, s)$ and $\nu$ be defined as in Theorem 4.2 .

Input: $A$ bounded pair $(\mathbf{a}, \mathbf{b})$ for $\pi$.

Output: $B T_{\pi}(\mathbf{a}, \mathbf{b})=\left(\left(\mathbf{a}^{\prime}, \mathbf{b}^{\prime}\right), k\right) \in \mathcal{X}(\pi)$.

(1) Let $t_{0}$ be the unique column containing the $\{r, s\}$-wire crossing in the rightlabeled wiring diagram for $\mathbf{a}$.

(2) Compute $\mathscr{B}_{t_{0}}^{-}(\mathbf{a}, \mathbf{b})=\left(\mathbf{a}^{\prime}, \mathbf{b}^{\prime}, i, j\right.$, outcome $)$.

(3) If outcome = deleted, then $j$ is the last crossing pushed in the bounded bumping algorithm before being deleted so $j \in[1, p]$. Return $\left(\left(\mathbf{a}^{\prime}, \mathbf{b}^{\prime}\right), j\right)$ and stop. By Proposition $2.7(3)$, we know $\left(\mathbf{a}^{\prime}, \mathbf{b}^{\prime}\right)$ is a bounded pair for $\nu$. 
(4) If outcome = bumped, return $\left(\left(\mathbf{a}^{\prime}, \mathbf{b}^{\prime}\right), 0\right)$ and stop. Proposition $2.7(2)$ shows that one of the wires crossing in column $j$ of the right-labeled wiring diagram for $\mathbf{a}^{\prime}$ is the $r$-wire, the other is labeled by some $q<r$ with $\ell(\pi)=\ell\left(\nu t_{q r}\right)$. Therefore, $\left(\mathbf{a}^{\prime}, \mathbf{b}^{\prime}\right)$ is a bounded pair for $\nu t_{q r}$.

Algorithm 4.4 (Inverse Bounded Transition). Suppose $\pi \neq \mathrm{id}$ is given, and let $(r, s)$ and $\nu$ be defined as in Theorem 4.2 .

Input: $((\mathbf{e}, \mathbf{f}), k) \in \mathcal{X}(\pi)$, in particular $(\mathbf{e}, \mathbf{f})$ is a bounded pair for $\nu t_{q, r}$ for some $1 \leqslant q \leqslant r$.

Output: $B T_{\pi}^{-1}((\mathbf{e}, \mathbf{f}), k)=(\mathbf{a}, \mathbf{b})$, a bounded pair for $\pi$.

(1) If $q=r$, then $k \in[1, p]$ and $\mathbf{e}=\left(e_{1}, \ldots, e_{p-1}\right) \in R(\nu)$. If $k<p$, set $\omega \leftarrow$ $s_{e_{p-1}} s_{e_{p-2}} \cdots s_{e_{k}}$, and if $k=p$ set $\omega \leftarrow$ id. Set

$$
\begin{aligned}
i & \leftarrow \omega^{-1}(r), \\
\mathbf{g} & \leftarrow \mathscr{I}_{k}^{i}(\mathbf{e}), \\
\mathbf{h} & \leftarrow \mathscr{I}_{k}^{0}(\mathbf{f}), \\
j & \leftarrow k .
\end{aligned}
$$

(2) If $q<r$, then $k=0$. Set $\mathbf{g} \leftarrow \mathbf{e}$ and $\mathbf{h} \leftarrow \mathbf{f}$. Let $j$ be the column of the $\{q, r\}$ wiring crossing in the right-labeled wiring diagram of $\mathbf{e}$ which must exist since $(\mathbf{e}, \mathbf{f}) \in \operatorname{BoundedPairs}\left(\nu t_{q, r}\right) \subset \mathcal{X}(\pi)$.

(3) Compute $\mathscr{B}_{j}^{+}(\mathbf{g}, \mathbf{h})=\left(\mathbf{g}^{\prime}, \mathbf{h}^{\prime}, i^{\prime}, t\right.$, bumped $)$. Return $\left(\mathbf{g}^{\prime}, \mathbf{h}^{\prime}\right)$ and stop. Note the outcome will always be bumped since we are applying increment-pushes. Lemma 4.5 below shows that in all cases, $\left(\mathbf{g}^{\prime}, \mathbf{h}^{\prime}\right)$ is a bounded pair for $\pi$.

Observe that $B T_{\pi}$ is an injection since the bounded bumping algorithm is reversible given the column $j$ of the final push and the value $r$ in the case outcome $=$ deleted. Thus, $B T_{\pi}^{-1} B T_{\pi}(\mathbf{a}, \mathbf{b})=(\mathbf{a}, \mathbf{b})$ so $B T_{\pi}(\mathbf{a}, \mathbf{b})=((\mathbf{e}, \mathbf{f}), j)=B T_{\pi}\left(\mathbf{a}^{\prime}, \mathbf{b}^{\prime}\right)$ implies $(\mathbf{a}, \mathbf{b})=\left(\mathbf{a}^{\prime}, \mathbf{b}^{\prime}\right)$. Also, $B T_{\pi}$ is surjective since $B T_{\pi}^{-1}$ is well defined on all of $\mathcal{X}(\pi)$ and one can observe that $B T_{\pi} B T_{\pi}^{-1}$ is again the identity map. Therefore, $B T_{\pi}$ : BoundedPairs $(\pi) \longrightarrow \mathcal{X}(\pi)$ is a bijection proving the Transition Equation for Bounded Pairs.

The following lemma is a generalization of Lemma 3.9.

LEMma 4.5. Assume the notation in Theorem 4.2. Let $\mathbf{a}=\left(a_{1}, \ldots, a_{p}\right)$ be any nearly reduced word at $1 \leqslant j \leqslant p$ such that $\mathscr{D}_{j}(\mathbf{a}) \in R(\nu)$ and the wires such that $\mathscr{D}_{j}(\mathbf{a}) \in$ $R(\nu)$ and the wires crossing in column $j$ of the right-labeled wiring diagram for a are labeled by $q<r$ where $r$ is the last descent of $\pi$. Let $\mathbf{b}$ be a bounded word for $\mathbf{a}$. If

$$
\mathscr{B}_{j}^{+}(\mathbf{a}, \mathbf{b})=\left(\mathbf{a}^{\prime}, \mathbf{b}^{\prime}, i^{\prime}, t, \text { bumped }\right),
$$

then $\left(\mathbf{a}^{\prime}, \mathbf{b}^{\prime}\right)$ is a bounded pair for $\pi$ and $t$ is the column containing the $\{r, s\}$-wire crossing in the right-labeled wiring diagram of $\mathbf{a}^{\prime}$.

Proof. By assumption, $\mathscr{D}_{j} \mathbf{a} \in R(\nu)$ so $\mathscr{D}_{t} \mathbf{a}^{\prime} \in R(\nu)$ by Proposition $2.7(3)$. Say wires $\{k, l\}$ with $k<l$ cross in column $t$ of $\mathbf{a}^{\prime}$. Then $\mathbf{a}^{\prime} \in R\left(\nu t_{k, l}\right)$.

By design, the $r$-wire is the larger labeled wire involved in the crossing in column $j$ of the right-labeled wiring diagram for a, hence the $r$ wire will continue to be one of the two wires crossed for every increment-push in the bounded bumping algorithm so $k=r<l$ by Proposition $2.7(2)$. Recall, $\nu(r)<\nu(s), \ell\left(\nu t_{r, s}\right)=\ell(\nu)+1$, and $(r, s)$ is the lex largest inversion of $\pi=\nu t_{r, s}$, so we know $\nu(m)<\nu(r)$ for every $m$ such that $r<m<s$. Thus, $l \geqslant s$. Furthermore, $\nu(s)<\nu(s+1)<\nu(s+2)<\ldots$, so the only possible value of $l$ such that $\ell\left(\nu t_{r, l}\right)=\ell(\nu)+1$ is $l=s$. Thus, we can conclude $\mathbf{a} \in R(\pi)$. 
Definition 4.6. Given $(\mathbf{a}, \mathbf{b}) \in \operatorname{BoundedPairs}(\pi)$, define the associated transition chain $Y^{\prime}(\mathbf{a}, \mathbf{b})$ recursively as follows. If $\pi=\mathrm{id}$, then $Y^{\prime}(\boldsymbol{a}, \boldsymbol{b}):=()$, the empty list. If $\pi \neq \mathrm{id}$, compute $B T_{\pi}(\mathbf{a}, \mathbf{b})=\left(\mathbf{a}^{\prime}, \mathbf{b}^{\prime}\right) \in \operatorname{BoundedPairs}\left(\pi t_{r, s} t_{q, r}\right) \subset \mathcal{X}(\pi)$ and $Y^{\prime}\left(\mathbf{a}^{\prime}, \mathbf{b}^{\prime}\right)=\left(\left(q_{k-1}, r_{k-1}\right), \ldots,\left(q_{2}, r_{2}\right),\left(q_{1}, r_{1}\right)\right)$. Prepend $(q, r)$ to get

$$
Y^{\prime}(\mathbf{a}, \mathbf{b}):=\left((q, r),\left(q_{k-1}, r_{k-1}\right), \ldots,\left(q_{2}, r_{2}\right),\left(q_{1}, r_{1}\right)\right) .
$$

Many bounded pairs for $\pi$ map to the same transition chain via $Y^{\prime}$. For example, when $\pi=[1,4,3,2]$ all 6 of the following bounded pairs map to $((1,3),(2,2),(2,2),(1,1))$ via $Y^{\prime}$.

\begin{tabular}{|c|c|}
\hline $\mathbf{a}$ & $\mathbf{b}$ \\
\hline 323 & 122 \\
323 & 123 \\
232 & 211 \\
232 & 212 \\
232 & 221 \\
232 & 231 \\
\hline
\end{tabular}

\section{Bijective Proof of MaCDOnALD's FORMula}

In this section, we spell out the promised bijection proving Macdonald's formula in Theorem 1.1. We introduce some notation first and then define the Macdonald map $M$ on all bounded pairs.

Recall from the introduction that both sides of the formula can be interpreted combinatorially. The sum on the left side of (1) clearly equals $|\operatorname{BoundedPairs}(\pi)|$. Let

$$
\mathcal{C}(\pi)=[1,1] \times[1,2] \times \cdots \times[1, p]
$$

where $p=\ell(\pi)$ and $[i, j]=\{i, i+1, \ldots, j\}$. Recall $\mathbf{c}=\left(c_{1}, c_{2}, \ldots, c_{p}\right) \in \mathcal{C}(\pi)$ is a substaircase word of length $p$. By Definition 2.8, one observes that the right side of (1) equals $|\mathcal{C}(\pi) \times \mathcal{R} \mathcal{P}(\pi)|$. We will refer to the elements $(\mathbf{c}, D) \in \cup_{\pi \in S_{\infty}} \mathcal{C}(\pi) \times \mathcal{R} \mathcal{P}(\pi)$ as $c D$-pairs for $\pi$.

We can now define a map $M$ from all bounded pairs to all $c D$-pairs which preserves the underlying permutation.

Algorithm 5.1 (Macdonald Map).

Input: $A$ bounded pair $(\mathbf{a}, \mathbf{b})=\left(\left(a_{1}, \ldots, a_{p}\right),\left(b_{1}, \ldots, b_{p}\right)\right)$. Let $\pi=s_{a_{1}} s_{a_{2}} \cdots s_{a_{p}}$. By definition of a bounded pair, a is reduced, so $p=\ell(\pi)$.

Output: $M(\mathbf{a}, \mathbf{b})=(\mathbf{c}, D) \in \mathcal{C}(\pi) \times \mathcal{R} \mathcal{P}(\pi)$.

(1) If $\pi$ is the identity, then we must have $(\mathbf{a}, \mathbf{b})=((),())$. Set $\mathbf{c}=()$ and $D=\{\}$. Return $(\mathbf{c}, D)$ and stop.

(2) Compute $B T_{\pi}(\mathbf{a}, \mathbf{b})=\left(\left(\mathbf{a}^{\prime}, \mathbf{b}^{\prime}\right), k\right) \in \mathcal{X}(\pi)$. Say $\left(\mathbf{a}^{\prime}, \mathbf{b}^{\prime}\right)$ is a bounded pair for $\nu t_{q, r}$ where $(r, s)$ is the lex largest inversion for $\pi, \nu=\pi t_{r, s}$ and $1 \leqslant q \leqslant r$.

(3) Recursively compute $M\left(\mathbf{a}^{\prime}, \mathbf{b}^{\prime}\right)=\left(\mathbf{c}^{\prime}, D^{\prime}\right)$. By induction on inversion order and Remark 2.11, we can assume that $\left(\mathbf{c}^{\prime}, D^{\prime}\right) \in \mathcal{C}\left(\nu t_{q, r}\right) \times \mathcal{R} \mathcal{P}\left(\nu t_{q, r}\right)$.

(4) Set $D=T_{\pi}^{-1}\left(D^{\prime}\right)$. If $q<r$, set $\mathbf{c}=\mathbf{c}^{\prime}$. Otherwise, if $q=r$, set $\mathbf{c}=\mathscr{I}_{p}^{k}\left(\mathbf{c}^{\prime}\right)$. Return $(\mathbf{c}, D)$ and stop. Observe that in either case, $\mathbf{c} \in \mathcal{C}(\pi)$. By Algorithm $3.3, D \in \mathcal{R} \mathcal{P}(\pi)$.

EXAmple 5.2. Consider the bounded pair $(\mathbf{a}, \mathbf{b})=((2,3,2),(2,1,2))$ for the permutation $\pi=[1,4,3,2]$. The steps from Algorithm 5.1 in this case are summarized in Table 6 . The result is $M((2,3,2),(2,1,2))=(\mathbf{c}, D)$ where $\mathbf{c}=(1,1,2)$ and $D$ is the pipe dream encoded by the biword $\left(\mathbf{r}_{D}, \mathbf{j}\right)=((2,3,2),(2,2,1))$. The transition chain is $Y(D)=((1,3),(2,2),(2,2),(1,1))$. Figure 1 from Section 1 illustrates the computations in this table using drawings of pipe dreams and wiring diagrams. 


\begin{tabular}{|c|c|c|c|c|}
\hline$\pi$ & $(\mathbf{a}, \mathbf{b})$ & $(q, r)$ & $k$ & $\left(\mathbf{c}, \mathbf{r}_{D}, \mathbf{j}_{D}\right)$ \\
\hline$[1432]$ & $(232,212)$ & $(1,3)$ & 0 & $(112,232,221)$ \\
{$[2413]$} & $(132,112)$ & $(2,2)$ & 2 & $(112,132,121)$ \\
{$[2314]$} & $(12,12)$ & $(2,2)$ & 1 & $(11,12,11)$ \\
{$[2134]$} & $(1,1)$ & $(1,1)$ & 1 & $(1,1,1)$ \\
{$[1234]$} & $(\varnothing, \varnothing)$ & & & $(\varnothing, \varnothing, \varnothing)$ \\
\hline
\end{tabular}

Figure 6 . Data for the computation $M((2,3,2),(2,1,2))$.

EXAmPLE 5.3. Consider the bounded pair $((5,4,3,5,6,4,5),(1,4,2,3,5,3,5))$ for $\pi=$ $[1,2,6,5,7,3,4]$. The steps from Algorithm 5.1 in this case are summarized in Table 7 . The result is the pair $(\mathbf{c}, D)=\left(\mathbf{c}, \mathbf{r}_{D}, \mathbf{j}_{D}\right)$ where $D$ is the pipe dream $D$ on the far left in Figure 5 and $\mathbf{c}=(1,1,1,3,2,1,3)$. Again, in the table, we use the biword encoding of pipe dreams, $\left(\mathbf{r}_{D}, \mathbf{j}_{D}\right)$.

\begin{tabular}{|c|c|c|c|c|}
\hline$\pi$ & $(\mathbf{a}, \mathbf{b})$ & $(q, r)$ & $k$ & $\left(\mathbf{c}, \mathbf{r}_{D}, \mathbf{j}_{D}\right)$ \\
\hline$[1265734]$ & $(5435645,1423535)$ & $(2,5)$ & 0 & $(1113213,4356435,4344212)$ \\
{$[146523]$} & $(5324534,1312424)$ & $(4,4)$ & 3 & $(1113213,324534,233211)$ \\
{$[146325]$} & $(523423,121313)$ & $(1,4)$ & 0 & $(111321,323543,322321)$ \\
{$[46135]$} & $(513423,111313)$ & $(3,3)$ & 1 & $(111321,312543,311321)$ \\
{$[24513]$} & $(13423,11313)$ & $(3,3)$ & 2 & $(11132,31243,31121)$ \\
{$[2431]$} & $(1323,1213)$ & $(3,3)$ & 3 & $(1113,3123,3111)$ \\
{$[2413]$} & $(132,122)$ & $(1,2)$ & 0 & $(111,312,311)$ \\
{$[3214]$} & $(121,111)$ & $(2,2)$ & 1 & $(111,212,211)$ \\
{$[3124]$} & $(21,11)$ & $(1,1)$ & 1 & $(11,21,21)$ \\
{$[2134]$} & $(1,1)$ & $(1,1)$ & 1 & $(1,1,1)$ \\
{$[1234]$} & $\varnothing, \varnothing)$ & & $\varnothing, \varnothing, \varnothing)$ \\
\hline
\end{tabular}

Figure 7. Data for the computation $M((5,4,3,5,6,4,5)$, $(1,4,2,3,5,3,5))$.

Proof of Theorem 1.1. Define $M_{\pi}$ to be the restriction of $M$ to BoundedPairs $(\pi)$. We will show by induction that $M_{\pi}$ is a bijection from $\operatorname{BoundedPairs}(\pi)$ to $\mathcal{C}(\pi) \times \mathcal{R} \mathcal{P}(\pi)$, as required to prove the theorem.

For the base case, if $\pi=$ id, then $M_{\text {id }}$ is the bijection mapping $((),()) \mapsto((),\{\})$. Now assume that $M_{\omega}$ : BoundedPairs $(\omega) \longrightarrow \mathcal{C}(\omega) \times \mathcal{R} \mathcal{P}(\omega)$ is a bijection for all permutations $\omega$ such that $\omega \prec \pi$, as in Remark 2.11.

By Theorem 4.2 , we know that $B T_{\pi}$ : $\operatorname{BoundedPairs}(\pi) \longrightarrow \mathcal{X}(\pi)$ is a bijection where

$$
\mathcal{X}(\pi)=(\text { BoundedPairs }(\nu) \times[1, p]) \cup \underset{\substack{q<r: \\ l(\pi)=l\left(\nu t_{q r}\right)}}{\bigcup} \text { BoundedPairs }\left(\nu t_{q r}\right) \times\{0\} .
$$

Let

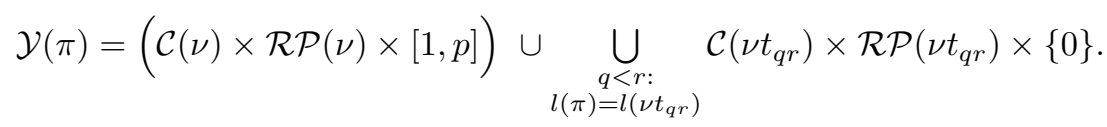

The induction hypothesis implies that the restricted map $M \times$ id $: \mathcal{X}(\pi) \longrightarrow \mathcal{Y}(\pi)$ is a bijection preserving the underlying permutation. That is, if $(\mathbf{a}, \mathbf{b}, k) \in \mathcal{X}(\pi)$, then 
$\mathbf{a} \in R\left(\nu t_{q, r}\right)$ for some $q \leqslant r$, and if $M \times \operatorname{id}(\mathbf{a}, \mathbf{b}, k)=(\mathbf{c}, D, k)$ then $D \in \mathcal{R} \mathcal{P}\left(\nu t_{q, r}\right)$ as well.

Define $R T_{\pi}: \mathcal{C}(\pi) \times \mathcal{R} \mathcal{P}(\pi) \longrightarrow \mathcal{Y}(\pi)$ by mapping

$$
R T_{\pi}(\mathbf{c}, D)= \begin{cases}\left(\widehat{\mathbf{c}}, T_{\pi}(D), c_{p}\right) & |D|>\left|T_{\pi}(D)\right| \\ \left(\mathbf{c}, T_{\pi}(D), 0\right) & |D|=\left|T_{\pi}(D)\right|,\end{cases}
$$

where $\widehat{\mathbf{c}}:=\left(c_{1}, \ldots, c_{p-1}\right)$. Since $T_{\pi}$ is a bijection, $R T_{\pi}$ is a bijection with well defined inverse $R T_{\pi}^{-1}: \mathcal{Y}(\pi) \longrightarrow \mathcal{C}(\pi) \times \mathcal{R} \mathcal{P}(\pi)$.

The map $M_{\pi}$ : BoundedPairs $(\pi) \longrightarrow \mathcal{C}(\pi) \times \mathcal{R} \mathcal{P}(\pi)$ can be written as the composition of three bijections, $M_{\pi}=R T_{\pi}^{-1} \circ(M \times \mathrm{id}) \circ B T_{\pi}$, hence is itself a bijection. This concludes the induction.

Observe from the proof above, we can show by induction that $M_{\pi}^{-1}=B T_{\pi}^{-1}$ 。 $\left(M^{-1} \times\right.$ id $) \circ R T_{\pi}$. Thus, we can write out the algorithm for $M^{-1}$ analogously with Algorithm 5.1.

Corollary 5.4. The inverse of $M$ is given by Algorithm 5.5.

Algorithm 5.5 (Inverse Macdonald Map).

Input: $(\mathbf{c}, D)$ where $D$ is a reduced pipe dream for some permutation $\pi$ and $\mathbf{c}=$ $\left(c_{1}, c_{2}, \ldots, c_{p}\right)$ is a sub-staircase word of length $p=\ell(\pi)$.

Output: $M^{-1}(\mathbf{c}, D)=(\mathbf{a}, \mathbf{b})$, a bounded pair for $\pi$.

(1) If $\pi$ is the identity, then we must have $\mathbf{c}=()$ and $D=\{\}$. Return $(\mathbf{a}, \mathbf{b})=$ $((),())$ and stop.

(2) Compute $T_{\pi}(D)=D^{\prime} \in \mathcal{U}(\pi)$. Say $D^{\prime}$ is a reduced pipe dream for $\nu t_{q, r}$ where $(r, s)$ is the lex largest inversion for $\pi, \nu=\pi t_{r, s}$ and $1 \leqslant q \leqslant r$. If $q<r$, set $k=0$ and $\mathbf{c}^{\prime}=\mathbf{c}$. Otherwise, if $q=r$, set $k=c_{p}$ and $\mathbf{c}^{\prime}=\left(c_{1}, \ldots, c_{p-1}\right)$.

(3) Recursively compute $M^{-1}\left(\mathbf{c}^{\prime}, D^{\prime}\right)=\left(\mathbf{a}^{\prime}, \mathbf{b}^{\prime}\right)$. By induction on inversion order and Remark 2.11, we can assume that $\left(\mathbf{a}^{\prime}, \mathbf{b}^{\prime}\right) \in \operatorname{BoundedPairs}\left(\nu t_{q, r}\right)$.

(4) Compute $B T_{\pi}^{-1}\left(\left(\mathbf{a}^{\prime}, \mathbf{b}^{\prime}\right), k\right)=(\mathbf{a}, \mathbf{b})$. Return $(\mathbf{a}, \mathbf{b})$ and stop. By the proof of Theorem 4.2, we know that $(\mathbf{a}, \mathbf{b})$ is a bounded pair for $\pi$.

REMARK 5.6. Observe that in step 2 of both Algorithm 5.1 and Algorithm 5.5, the data $(q, r)$ and $k$ are determined from the input. So if $M(\mathbf{a}, \mathbf{b})=(\mathbf{c}, D)$, then by step 4 of both algorithms, these 3 quantities, $k, q, r$ are the same. In particular, $Y(D)=$ $Y^{\prime}(\mathbf{a}, \mathbf{b})$.

REMARK 5.7. In general, it is not easy to "eyeball" the map $M$ or $M^{-1}$ by simply straightening out or bending wires without passing through the transition chain. Every biword coming from a reduced pipe dream for $\pi$ is a bounded pair for $\pi$, but the converse does not hold. Thus, the map $M^{-1}$ rarely acts as the identity map. In fact, we know $M$ is a $p$ ! to 1 map if we project the image onto the pipe dreams.

\section{6. q-ANALOGUE OF MACDONALD'S FORMULA}

In this section, we prove Theorem 1.2 bijectively. The first step is to rewrite the left side of (2) as a specialization of the bounded pair polynomial $\mathfrak{F}_{\pi}(\mathbf{x}, \mathbf{y} ; \mathfrak{q})$ defined in Definition 4.1. We then prove that this specialized polynomial satisfies a $\mathfrak{q}$-analog of the Transition Equation for Bounded Pairs, and thus argue that every step of our algorithmic bijection $B T_{\pi}$ respects the $\mathfrak{q}$-weight so $M$ is a $\mathfrak{q}$-weight preserving bijection in addition to preserving the underlying permutation.

Specializing each $x_{i}=\mathfrak{q}$ and $y_{i}=\mathfrak{q}^{-1}$ in $\mathfrak{F}_{\pi}(\mathbf{x}, \mathbf{y} ; \mathfrak{q})$, where the third parameter is the same formal variable $\mathfrak{q}$, gives a one parameter version of the bounded pair 
polynomial

$$
f_{\pi}(\mathfrak{q}):=\sum \mathfrak{q}^{(\mathbf{a}, \mathbf{b})}=\sum_{\left(a_{1}, a_{2}, \ldots, a_{p}\right) \in R(\pi)} \mathfrak{q}^{\operatorname{comaj}(\mathbf{a})}\left[a_{1}\right] \cdot\left[a_{2}\right] \cdots\left[a_{p}\right]
$$

where the first sum is over all bounded pairs $(\mathbf{a}, \mathbf{b})$ for $\pi$ and $\mathfrak{q}^{(\mathbf{a}, \mathbf{b})}$ is the combined weight

$$
\mathfrak{q}^{(\mathbf{a}, \mathbf{b})}:=\mathfrak{q}^{\operatorname{comaj}(\mathbf{a})} \prod_{i=1}^{p} \mathfrak{q}^{a_{i}-b_{i}} .
$$

For example, let $s_{r}$ be a simple transposition for some $r \geqslant 1$. Then $f_{s_{r}}(\mathfrak{q})=[r]=$ $1+\mathfrak{q}+\mathfrak{q}^{2}+\ldots+\mathfrak{q}^{r-1}$. See also the example after Theorem 1.2.

Theorem 6.1 (q-Transition Equation for Bounded Pairs). For all permutations $\pi$ such that $\ell(\pi)=p>0$, the polynomials $f_{\pi}(\mathfrak{q})$ satisfy the following recursive formula:

$$
f_{\pi}(\mathfrak{q})=\left(1+\mathfrak{q}+\cdots+\mathfrak{q}^{p-1}\right) \mathfrak{q}^{r-1} f_{\nu}(\mathfrak{q})+\sum_{\substack{q<r \\ l(\pi)=l\left(\nu t_{q r}\right)}} f_{\nu t_{q r}}(\mathfrak{q})
$$

where $(r, s)$ is the lex largest inversion in $\pi$, and $\nu=\pi t_{r s}$. The base case of the recurrence is $f_{\text {id }}(\mathfrak{q})=1$.

A bijective proof of Theorem 6.1 implies a bijective proof of Theorem 1.2 since $f_{\pi}(\mathfrak{q})$ is by definition the left side of (2), while the right side satisfies the same recurrence and base conditions as (10) by the Transition Equation for Schubert polynomials, Theorem 2.9.

To prove Theorem 6.1, we need to understand how the maps $B T_{\pi}$ and $B T_{\pi}^{-1}$ change the combined weight for bounded pairs. This involves an investigation of the comaj statistic on reduced words. Recall for motivation the well known formula due to MacMahon relating the number of inversions to the comaj statistic

$$
\left(1+\mathfrak{q}+\cdots+\mathfrak{q}^{n-1}\right) \sum_{\nu \in S_{n-1}} \mathfrak{q}^{\ell(\nu)}=\sum_{\pi \in S_{n}} \mathfrak{q}^{\ell(\pi)}=\sum_{\pi \in S_{n}} \mathfrak{q}^{\operatorname{comaj}(\pi)}
$$

The first equality follows simply by inserting $n$ into the one-line notation for $\nu \in$ $S_{n-1}$ and observing the change in the number of inversions. The second equality can similarly be proved using the code of a permutation and the Carlitz bijection [5], see also [35, 43]. Note, the Carlitz bijection is different than Foata's famous bijective proof of the second equality [8].

We next state a mild generalization of a lemma due to Gupta [15] about how comaj changes when one additional letter is inserted into a word in every possible way. Gupta's proof covers the case where the numbers $a_{k}$ are all distinct. Lemma 6.2 below extends this to sequences with no two adjacent values equal. We include a short proof of Gupta's lemma below as a prelude to extending this analysis to reduced words in Lemma 6.4 and the proof of Theorem 6.1. For another proof and further applications of "insertion lemmas" in the literature, see $[16,36]$.

Fix any sequence of real numbers $\mathbf{a}=\left(a_{1}, \ldots, a_{p}\right)$. For $1 \leqslant i \leqslant p+1$, let

$$
\mathbf{a}_{i}^{j}:=\mathscr{I}_{i}^{j}(\mathbf{a})=\left(a_{1}, \ldots, a_{i-1}, j, a_{i}, \ldots, a_{p}\right)
$$

be the result of inserting $j$ into a to become column $i$. We also extend the definition of the comajor index to arbitrary real sequences: $\operatorname{comaj}(\mathbf{a}):=\sum_{i: a_{i}<a_{i+1}} i$.

Lemma 6.2 ([15]). Fix any sequence of real numbers $\mathbf{a}=\left(a_{1}, \ldots, a_{p}\right)$ such that no two adjacent elements $a_{i}, a_{i+1}$ are equal, and let $j$ be a real number different from $\left\{a_{1}, \ldots, a_{p}\right\}$. Then, we have

$$
\left\{\operatorname{comaj}\left(\mathbf{a}_{i}^{j}\right)-\operatorname{comaj}(\mathbf{a}): 1 \leqslant i \leqslant p+1\right\}=\{0,1,2, \ldots, p\} .
$$


For example, take $\mathbf{a}=(2,3,5,2)$ and $j=4$. Then $\operatorname{comaj}(\mathbf{a})=3$. The five words obtained from a by inserting 4 in all columns are given below with their comaj.

\begin{tabular}{|c|c|c|c|}
\hline$i$ & $\mathbf{a}_{i}^{4}$ & $\operatorname{comaj}\left(\mathbf{a}_{i}^{4}\right)$ & $\operatorname{comaj}\left(\mathbf{a}_{i}^{4}\right)-\operatorname{comaj}(\mathbf{a})$ \\
\hline 1 & 42352 & 5 & 2 \\
2 & 24352 & 4 & 1 \\
3 & 23452 & 6 & 3 \\
4 & 23542 & 3 & 0 \\
5 & 23524 & 7 & 4 \\
\hline
\end{tabular}

As one can see, the difference in comaj takes on the five values from 0 to 4 in permuted order. We refer to the word $(2,1,3,0,4)$ as the comaj difference word.

The conclusion of the lemma does not hold when adjacent equal elements are allowed even if we extend the definition of comaj to cover weak ascents. For example, if $\mathbf{a}=(1,1)$ and $j=1$, then $\operatorname{comaj}\left(\mathbf{a}_{i}^{j}\right)-\operatorname{comaj}(\mathbf{a})$ is constant for all $i=0,1,2$.

Proof. The statement is easily checked for $p=0,1$. Let $k$ be a real number distinct from $\left\{j, a_{p}\right\}$, and let $\mathbf{a}^{\prime}=\left(a_{1}, \ldots, a_{p}, k\right)$. Assume by induction on $p \geqslant 1$ that the statement holds for $\mathbf{a}$, so we can assume $\left\{\operatorname{comaj}\left(\mathbf{a}_{i}^{j}\right)-\operatorname{comaj}(\mathbf{a}): 1 \leqslant i \leqslant p+1\right\}=$ $\{0,1,2, \ldots, p\}$.

The final element in the comaj difference word for a can be determined from the relative order of $a_{p}, j$. If $a_{p}<j$, then $\operatorname{comaj}\left(\mathbf{a}_{p+1}^{j}\right)-\operatorname{comaj}(\mathbf{a})=p$ and if $a_{p}>j$, then $\operatorname{comaj}\left(\mathbf{a}_{p+1}^{j}\right)-\operatorname{comaj}(\mathbf{a})=0$.

Next, consider the relative order of $a_{p}, j, k$ and how it affects the comaj difference word. The possible orders correspond with the 6 permutations in $S_{3}$. For instance, if $j<a_{p}<k$, then $\operatorname{comaj}\left(\mathbf{a}_{i}^{\prime j}\right)-\operatorname{comaj}\left(\mathbf{a}^{\prime}\right)=\operatorname{comaj}\left(\mathbf{a}_{i}^{j}\right)-\operatorname{comaj}(\mathbf{a})+1$ for all $1 \leqslant i \leqslant p$ since $k$ adds one new ascent to the right of all these columns which gets shifted over when $j$ is inserted. Since $j<a_{p}, \operatorname{comaj}\left(\mathbf{a}_{p+1}^{j}\right)-\operatorname{comaj}(\mathbf{a})=0$ as noted above so $\left\{\operatorname{comaj}\left(\mathbf{a}_{i}^{\prime j}\right)-\operatorname{comaj}\left(\mathbf{a}^{\prime}\right): 1 \leqslant i \leqslant p\right\}=\{2, \ldots, p+1\}$ by the induction hypothesis. Furthermore, since $j<a_{p}<k, \operatorname{comaj}\left(\mathbf{a}_{p+1}^{\prime j}\right)-\operatorname{comaj}\left(\mathbf{a}^{\prime}\right)=1$ and $\operatorname{comaj}\left(\mathbf{a}_{p+2}^{\prime j}\right)-\operatorname{comaj}\left(\mathbf{a}^{\prime}\right)=0$. Thus, the claim holds for $\mathbf{a}^{\prime}$ in this case as well.

Each of the remaining 5 cases is similar. They only depend on the relative order of $a_{p}, k, j$ and not on any of the specific values $a_{1}, \ldots, a_{p}, j, k$. We leave the remaining cases to the reader or their computer to check.

We can now use Lemma 6.2 to give a bijective proof of MacMahon's formula, Equation (11). A similar argument is implicit in [5].

Corollary 6.3. For all $n \geqslant 2$,

$$
\sum_{\pi \in S_{n}} \mathfrak{q}^{\operatorname{comaj}(\pi)}=\left(1+\mathfrak{q}+\cdots+\mathfrak{q}^{n-1}\right) \sum_{\nu \in S_{n-1}} \mathfrak{q}^{\operatorname{comaj}(\nu)} .
$$

Proof. For each permutation $\nu \in S_{n-1}$ written in one-line notation, there are $n$ ways to insert $n$. By Lemma 6.2 , the comaj statistic will increase by a distinct value in $\{0,1, \ldots, n-1\}$ for each of these ways.

Next we prove a variation of Lemma 6.2 involving reduced words. The idea is similar to the proof of Lemma 6.2, though the proof is much more technical.

Given a reduced word $\mathbf{a}=\left(a_{1}, \ldots, a_{p}\right)$, draw its left-labeled wiring diagram, as in the first diagram of Figure 8. Fix a positive integer $j$ and consider the $j$-wire. Let $h_{i}^{j}(\mathbf{a})$ be the row of the $j$-wire in column $i-1$, so $h_{i}^{j}(\mathbf{a})=s_{a_{i-1}} \cdots s_{a_{2}} s_{a_{1}}(j)$. In the notation of Section $2.2, h_{i}^{j}(\mathbf{a})=\pi_{i-1}^{-1}(j)$, where $\pi_{t}$ denotes the permutation at time $t$ 

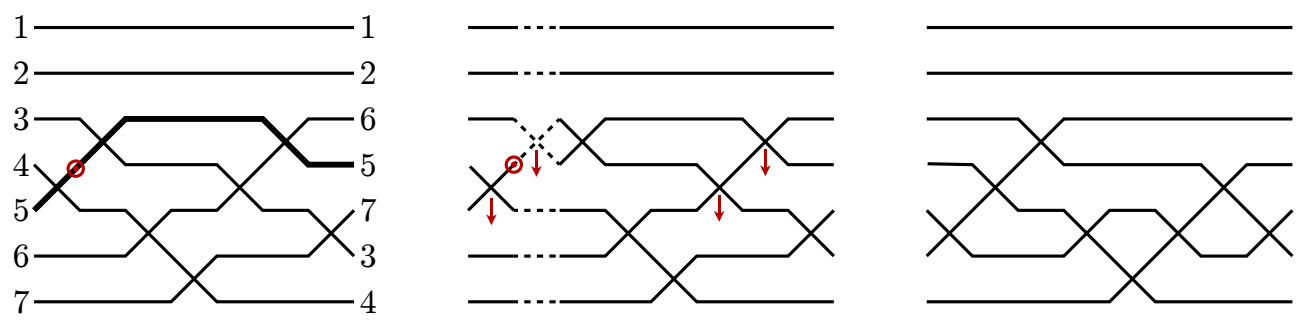

Figure 8. An example of the transformation from a (left) to $\widetilde{\mathbf{a}}$ (middle) to $\widetilde{\mathbf{a}}^{\prime}$ (right), with $i=2$ and $j=5$. The row of wire 5 just before the 2 nd crossing is $h_{2}^{5}(\mathbf{a})=4$, so we insert a crossing on row 3 in such a way as to become column 2 . Then we apply an increment-bump to this crossing to obtain $\widetilde{\mathbf{a}}^{\prime}$.

of a. We insert a new crossing in column $i$ with its left foot meeting the $j$-wire; i.e. define

$$
\widetilde{\mathbf{a}}:=\mathscr{I}_{i}^{h_{i}^{j}(\mathbf{a})-1}(\mathbf{a})=\left(a_{1}, a_{2}, \ldots, a_{i-1}, h_{i}^{j}(\mathbf{a})-1, a_{i}, \ldots, a_{p}\right)
$$

Then, $\widetilde{\mathbf{a}}$ may or may not be a reduced word itself, but it is nearly reduced at $i$. Now we want to apply a Little bump to $\widetilde{\mathbf{a}}$. To be consistent with our earlier definitions, we write this in terms of bounded bumping: every word is a bounded word for itself, so we can apply the bounded bumping algorithm with input $(\widetilde{\mathbf{a}}, \widetilde{\mathbf{a}}, i,+)$. Say $\mathscr{B}_{i}^{+}(\widetilde{\mathbf{a}}, \widetilde{\mathbf{a}})=$ $\left(\widetilde{\mathbf{a}}^{\prime}, \widetilde{\mathbf{b}}^{\prime}, g, h\right.$, outcome). Set

$$
y_{i}^{j}(\mathbf{a}):=\widetilde{\mathbf{a}}^{\prime}
$$

Define the augmented comaj difference word for a along wire $j$ to be $\mathbf{v}^{j}(\mathbf{a}):=$ $\left(v_{1}^{j}(\mathbf{a}), \ldots, v_{p+1}^{j}(\mathbf{a})\right)$ where

$$
v_{i}^{j}(\mathbf{a})=\operatorname{comaj}\left(y_{i}^{j}(\mathbf{a})\right)-\operatorname{comaj}(\mathbf{a})+h_{i}^{j}(\mathbf{a})-1 .
$$

The augmented comaj difference word measures the change in the power of $\mathfrak{q}$ in the combined weight when the $B T_{\pi}$ algorithm ends in a deletion in each column of step $2(\mathrm{a})$.

Consider the running example of the reduced word $\mathbf{a}=(4,3,5,6,4,3,5)$, and fix $j=$ 5 . We compute comaj $(\mathbf{a})=11$. The wiring diagram for $\mathbf{a}$ is shown in the first diagram of Figure 8 . Observe that the row of the 5 -wire in the wiring diagram decreases to 3 and then increases to 4 in matrix coordinates. In the second diagram in Figure 8 , we show the wiring diagram for $\widetilde{a}=(4,3,3,5,6,4,3,5)$ computed by inserting an extra crossing in the second column with its left foot on the 5 -wire, so inserting a 3 into a. The arrows indicate pushes in the bounded bumping algorithm for $\mathscr{B}_{2}^{+}(\widetilde{\mathbf{a}}, \widetilde{\mathbf{a}})$; they occur in columns $2,1,7,6$ in sequential order. The third diagram shows the wiring diagram of $y_{2}^{5}(\mathbf{a})=\widetilde{\mathbf{a}}^{\prime}=(5,4,3,5,6,5,4,5)$. Compute $\operatorname{comaj}\left(y_{2}^{5}(\mathbf{a})\right)=14$, so $v_{2}^{5}(\mathbf{a})=$ $14-11+3=6$. Next we display the data to compute the augmented comaj difference 
word for $\mathbf{a}$.

\begin{tabular}{|c|c|c|c|c|c|c|}
\hline$i$ & $h_{i}^{5}(\mathbf{a})$ & insert & $y_{i}^{5}(\mathbf{a})$ & $\operatorname{comaj}\left(y_{i}^{5}(\mathbf{a})\right.$ & $h_{i}^{5}(\mathbf{a})-1$ & $v_{i}^{5}(\mathbf{a})$ \\
\hline 1 & 5 & 44356435 & 54356545 & 14 & 4 & 7 \\
2 & 4 & 43356435 & 54356545 & 14 & 3 & 6 \\
3 & 3 & 43256435 & 54356545 & 14 & 2 & 5 \\
4 & 3 & 43526435 & 54536545 & 13 & 2 & 4 \\
5 & 3 & 43562435 & 54563545 & 17 & 2 & 8 \\
6 & 3 & 43564235 & 54565345 & 18 & 2 & 9 \\
7 & 4 & 43564335 & 54565345 & 18 & 3 & 10 \\
8 & 4 & 43564353 & 43564354 & 11 & 3 & 3 \\
\hline
\end{tabular}

Thus, $v^{5}(\mathbf{a})=(7,6,5,4,8,9,10,3)$. Further examples appear in the Appendix which demonstrates the computation of $\mathbf{v}^{5}$ (a) for all the initial substrings of $(4,3,5,6,4,3,5)$.

Lemma 6.4. Given a reduced word $\mathbf{a}=\left(a_{1}, \ldots, a_{p}\right)$ and a fixed positive integer $j$, the augmented comaj difference word $\mathbf{v}^{j}(\mathbf{a})=\left(v_{1}^{j}(\mathbf{a}), \ldots, v_{p+1}^{j}(\mathbf{a})\right)$ is a permutation of the integers in the closed interval $\left[h_{p+1}^{j}(\mathbf{a})-1, h_{p+1}^{j}(\mathbf{a})+p-1\right]$. Moreover, every entry of $\mathbf{v}^{j}(\mathbf{a})$ is a record, i.e. it is either greater than all preceding entries or less than all preceding entries.

The proof is by induction based on initial substrings of $\mathbf{a}$, similar to the proof of Lemma 6.2. It is complicated by the fact that we are inserting a crossing along the $j$-wire so the value which we are inserting varies with column.

Proof. We prove the statement by induction on $p$. The statement is true for the empty reduced word since $\mathbf{v}^{j}()=(j-1)$. There are 4 cases to check if $p=1$. Say $\mathbf{a}=\left(a_{1}\right)$, so $h_{1}^{j}(\mathbf{a})=j$ and $h_{2}^{j}(\mathbf{a})=s_{k}(j)$. Then,

$$
\mathbf{v}^{j}\left(\left(a_{1}\right)\right)= \begin{cases}(j, j-1) & j<a_{1} \\ (j, j+1) & j=a_{1} \\ (j-1, j-2) & j=a_{1}+1 \\ (j-1, j) & j>a_{1}+1 .\end{cases}
$$

Thus, all 4 cases for $p=1$ satisfy the statements in the lemma.

Assume the lemma holds by induction for all reduced words up to length $p \geqslant 1$. We will show it holds for all reduced words of length $p+1$. Let

$$
\mathbf{a}^{\prime}=\left(a_{1}, \ldots, a_{p}, k\right)
$$

be a reduced word extending $\mathbf{a}$. Thus, $h_{i}^{j}\left(\mathbf{a}^{\prime}\right)=h_{i}^{j}(\mathbf{a})$ for $1 \leqslant i \leqslant p+1$. Let

$$
h=h_{p+1}^{j}(\mathbf{a})=s_{a_{p}} \cdots s_{a_{1}}(j)
$$

then $h_{p+2}^{j}\left(\mathbf{a}^{\prime}\right)=s_{k}(h)$.

Our goal is to compute the augmented comaj differences $v_{i}^{j}\left(\mathbf{a}^{\prime}\right)$ for $1 \leqslant i \leqslant p+2$. We will treat the three cases $1 \leqslant i \leqslant p, i=p+1$ and $i=p+2$ separately.

First consider the case $1 \leqslant i \leqslant p$. The bounded bumping algorithm preserves the ascent set of a word by Proposition 2.7(4), so one can observe that

$$
\operatorname{comaj}\left(y_{i}^{j}(\mathbf{a})\right)=\operatorname{comaj}\left(a_{1}, a_{2}, \ldots, a_{i-1}, h_{i}^{j}(\mathbf{a})-1 / 2, a_{i}, \ldots, a_{p}\right) .
$$

This fact will allow us to compute the augmented comaj differences without knowing the exact sequence of pushes required in the bounded bumping algorithm. Using the above observation,

$$
\operatorname{comaj}\left(y_{i}^{j}\left(\mathbf{a}^{\prime}\right)\right)=\operatorname{comaj}\left(y_{i}^{j}(\mathbf{a})\right)+(p+1) \cdot \delta,
$$


while

$$
\operatorname{comaj}\left(\mathbf{a}^{\prime}\right)=\operatorname{comaj}(\mathbf{a})+p \cdot \delta
$$

where

$$
\delta:= \begin{cases}1, & a_{p}<k \\ 0, & \text { otherwise. }\end{cases}
$$

Since also $h_{i}^{j}\left(\mathbf{a}^{\prime}\right)=h_{i}^{j}(\mathbf{a})$, we conclude by combining the last three equations that

$$
v_{i}^{j}\left(\mathbf{a}^{\prime}\right)=v_{i}^{j}(\mathbf{a})+\delta
$$

We compute $v_{p+1}^{j}(\mathbf{a})=\operatorname{comaj}\left(y_{p+1}^{j}(\mathbf{a})\right)-\operatorname{comaj}(\mathbf{a})+h_{p+1}^{j}(\mathbf{a})-1=p \cdot \delta+h-1$. By induction, we know $\left(v_{1}^{j}(\mathbf{a}), \ldots, v_{p}^{j}(\mathbf{a})\right)$ is a permutation with every entry being a record of the interval $[h-1, h+p-1] \backslash\{p \cdot \delta+h-1\}$. Therefore $\left(v_{1}^{j}\left(\mathbf{a}^{\prime}\right), \ldots, v_{p}^{j}\left(\mathbf{a}^{\prime}\right)\right)$ is in fact a permutation of an interval of consecutive integers such that every entry is a record.

Now we consider the case $i=p+1$. We claim that the value $v_{p+1}^{j}\left(\mathbf{a}^{\prime}\right)$ is completely determined by the values $h, a_{p}, k, p$ as follows. Note that $a_{p}$ exists since $p \geqslant 1$ by assumption, and $a_{p} \neq k$ since they are adjacent in a reduced word. All possible cases are

$$
v_{p+1}^{j}\left(\mathbf{a}^{\prime}\right)= \begin{cases}h+p & a_{p}>k \geqslant h \\ h-1 & a_{p}<k<h \\ p \cdot \delta+h-1+\delta & \text { otherwise. }\end{cases}
$$

We conclude here that $\left(v_{1}^{j}\left(\mathbf{a}^{\prime}\right), \ldots, v_{p+1}^{j}\left(\mathbf{a}^{\prime}\right)\right)$ is in fact a permutation of an interval of consecutive integers such that every entry is a record. In the case $a_{p}>k \geqslant h$, the interval is $[h, h+p]$, and in the other two cases the interval is $[h-1, h+p-1]$.

Finally, consider the case $i=p+2$. Again, the value $v_{p+2}^{j}\left(\mathbf{a}^{\prime}\right)$ is straightforward to calculate from the definition of the augmented comaj vector given $k$ and the fact that $s_{k}(h)=h_{p+2}^{j}\left(\mathbf{a}^{\prime}\right)$ mentioned above:

$$
v_{p+2}^{j}\left(\mathbf{a}^{\prime}\right)= \begin{cases}s_{k}(h)-1 & k \geqslant s_{k}(h) \\ p+s_{k}(h) & k<s_{k}(h) .\end{cases}
$$

Thus, $v_{p+2}^{j}\left(\mathbf{a}^{\prime}\right)$ will be an extreme value in the interval $\left[s_{k}(h)-1, s_{k}(h)+p\right]$ as required for the lemma. All that remains to prove the lemma is to ascertain how $v_{p+2}^{j}\left(\mathbf{a}^{\prime}\right)$ relates to $[h, h+p]$ when $a_{p}>k \geqslant h$ or $[h-1, h+p-1]$ otherwise. This again breaks into cases depending on if $s_{k}(h)=h, h-1, h+1$. We leave this straightforward verification to the reader.

Proof of Theorem 6.1. As mentioned in the introduction to this section, we will show that the bijection $B T_{\pi}$ from Algorithm 4.3 preserves the $\mathfrak{q}$-weight in the following sense. Assume $B T_{\pi}(\mathbf{a}, \mathbf{b})=((\mathbf{e}, \mathbf{f}), k) \in \mathcal{X}(\pi)$. Let $j=\pi(r)$ so that $h_{p+1}^{j}(\mathbf{a})=r$. We will show that the combined weight defined in (9) satisfies

$$
\mathfrak{q}^{(\mathbf{a}, \mathbf{b})}= \begin{cases}\mathfrak{q}^{v_{k}^{j}(\mathbf{e})} \mathfrak{q}^{(\mathbf{e}, \mathbf{f})} & k>0 \\ \mathfrak{q}^{(\mathbf{e}, \mathbf{f})} & k=0 .\end{cases}
$$

Once this is complete, we know from Lemma 6.4 that $v^{j}(\mathbf{a})$ is a permutation of $[r-1, r+p-1]$. Hence, Theorem 6.1 follows by a straightforward verification.

Every $((\mathbf{e}, \mathbf{f}), k) \in \mathcal{X}(\pi)$ corresponds with a pair $q \leqslant r$ such that $(\mathbf{e}, \mathbf{f})$ is a bounded pair for $\nu t_{q, r}$. Recall, $k=0$ if and only if $q<r$.

In the case $k=0$, the combined weight is preserved since the bounded bumping algorithm preserves the ascent set of a word by Proposition $2.7(4)$. Furthermore, the 
differences $a_{i}-b_{i}$ for all $i$ are preserved by every push step in the bounded bumping algorithm.

When $k>0,(\mathbf{e}, \mathbf{f})$ is a bounded pair for $\nu$. The computation for $B T_{\pi}(\mathbf{a}, \mathbf{b})$ removed a letter from column $k$ on the last step. The crossing removed had its right foot on the wire labeled $r$ in the right-labeled diagram for e, or equivalently the wire labeled $j=\pi(r)$ when the diagram is labeled increasing along the left side. Therefore, the row of the removed crossing is $h_{k}^{j}(\mathbf{e})-1$. We also must have $y_{k}^{j}(\mathbf{e})=\mathbf{a}$ by definition of the $y_{k}^{j}$ map and the fact that the bounded bumping algorithm is reversible by Proposition 2.7(1). So $h_{k}^{j}(\mathbf{e})-1=a_{k}-b_{k}$. In all columns $i \neq k$, the difference $a_{i}-b_{i}$ is preserved by every push step in the bounded bumping algorithm. Using the notation

$$
v_{k}^{j}(\mathbf{e})=\operatorname{comaj}(\mathbf{a})-\operatorname{comaj}(\mathbf{e})+h_{k}^{j}(\mathbf{e})-1,
$$

we have shown $\mathfrak{q}^{(\mathbf{a}, \mathbf{b})}=\mathfrak{q}^{v_{k}^{j}(\mathbf{e})} \mathfrak{q}^{(\mathbf{e}, \mathbf{f})}$.

\section{FOMIN-Kirillov Formulas}

Fomin and Kirillov [11] gave several identities generalizing Macdonald's formula, and posed the problem of finding bijective proofs. We show that our bijection implies a bijective proof of one of these identities involving dominant permutations. We first state the identity, starting with an important special case. In the interest of brevity, we will assume the reader has some familiarity with plane partitions and standard Young tableaux. More information on these objects may be found in the cited references.

Let $w_{0}=[n, n-1, \ldots, 1] \in S_{n}$. The following formula specializes to Macdonald's formula (1) when $x=0$ and the coefficient of the leading term is $\# R\left(w_{0}\right)$. The last quantity equals the number of standard Young tableaux of staircase shape with $n-1$ rows, as proved by Stanley [44], and later bijectively by Edelman and Greene [7].

THEOREM 7.1 ([11, Theorem 1.1]). We have the following identity of polynomials in $x$ for the permutation $w_{0} \in S_{n}$ :

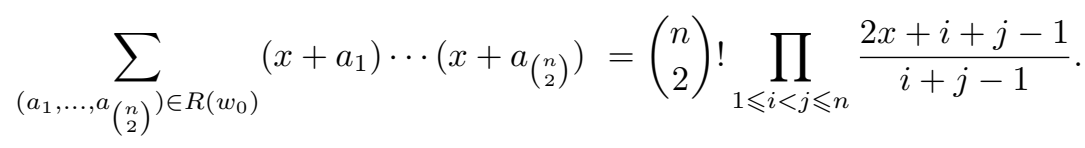

The second factor on the right side of (13) counts the number of plane partitions with maximum entry $x$. For a permutation $\pi=[\pi(1), \ldots, \pi(n)] \in S_{n}$, write $1^{x} \times$ $\pi=[1,2, \ldots, x, \pi(1)+x, \pi(2)+x, \ldots, \pi(n)+x]$. Via theorems of Wachs [47] and Proctor $[38,39,23]$, the second factor on the right of $(13)$ is also the number of terms in the Schubert polynomial for $1^{x} \times w$ when $x$ is a nonnegative integer. A bijection between the sets $R\left(1^{x} \times \pi\right)$ and $R(\pi)$ is given by $\left(a_{1}, a_{2}, \ldots, a_{p}\right) \mapsto\left(x+a_{1}, x+\right.$ $\left.a_{2}, \ldots, x+a_{p}\right)$.

Fomin and Kirillov gave a $\mathfrak{q}$-analog of the above identity in which, moreover, $w_{0}$ is generalized to an arbitrary dominant permutation. A dominant permutation is one whose code is weakly decreasing. For any partition $\lambda \vdash p$, let $\sigma_{\lambda}$ be the dominant permutation in $S_{p+1}$ whose code is $\lambda$ followed by zeros. Let $\operatorname{rpp}^{\lambda}(x)$ be the set of weak reverse plane partitions whose entries are all in the range $[0, x]$ for $x \in \mathbb{N}$. This is the set of $x$-bounded fillings of $\lambda$ with rows and columns weakly increasing to the right and down. Given a weak reverse plane partition $P$, let $|P|$ be the sum of its entries. Let

$$
\left[r p p^{\lambda}(x)\right]_{\mathfrak{q}}=\sum_{P \in r p p^{\lambda}(x)} \mathfrak{q}^{|P|}
$$


Theorem $7.2([11$, Theorem 3.1]). For any partition $\lambda \vdash p$ and its associated dominant permutation $\sigma_{\lambda}$, we have the following identity for all $x \in \mathbb{N}$ :

$$
\begin{aligned}
\sum_{\left(a_{1}, a_{2}, \ldots, a_{p}\right) \in R\left(\sigma_{\lambda}\right)} \mathfrak{q}^{\operatorname{comaj}\left(a_{1}, a_{2}, \ldots, a_{p}\right)} & {\left[x+a_{1}\right] \cdot\left[x+a_{2}\right] \cdots\left[x+a_{p}\right] } \\
& =[p] ! \mathfrak{S}_{1^{x} \times \sigma_{\lambda}}\left(1, \mathfrak{q}, \mathfrak{q}^{2}, \ldots, \mathfrak{q}^{x+p}\right) \\
& =[p] ! \mathfrak{q}^{b(\lambda)}\left[r p p^{\lambda}(x)\right]_{\mathfrak{q}}
\end{aligned}
$$

where $b(\lambda)=\sum_{i}(i-1) \lambda_{i}$.

The first equality is given by Macdonald's q-formula. The second follows from the theorem of Wachs [47] proving that for every vexillary permutation $\pi$, its Schubert polynomial is a flagged Schur function of shape determined by sorting the Lehmer code of $\pi$. (Dominant permutations are vexillary). Using our bijection for Macdonald's formula, we can now give a complete bijective proof of Theorem 7.2 as requested in [11, Open Problem 1].

Proof. Fix a partition $\lambda$ and $x \in \mathbb{N}$. We construct a bijection $F K$ from bounded pairs for $\sigma_{\lambda}$ to the set of sub-staircase words of length $|\lambda|$ times the set of reverse plane partitions for $\lambda$ bounded by $x$ as follows.

(1) Given a bounded pair $(\mathbf{a}, \mathbf{b})$ for $\sigma_{\lambda}$, let $(\mathbf{c}, D)=M(\mathbf{a}, \mathbf{b})$ be the corresponding $c D$-pair using the Macdonald Map specified in Section 5.

(2) From $D$, read the vectors of row numbers $\mathbf{i}_{D}=\left(i_{1}, \ldots, i_{p}\right)$ and diagonal numbers $\mathbf{r}_{D}$, as described in Section 3. (Note the contrast with earlier proofs, where we used column numbers; the vector $\mathbf{i}_{D}$ is sometimes called a compatible sequence for $\mathbf{r}_{D}$ - see [3].)

(3) Let $\left(P_{D}, Q_{D}\right)$ be the insertion tableau and recording tableau of the EdelmanGreene bijection [7] applied to the reduced word $\mathbf{r}_{D}$. Let $\left(P_{D}^{T}, Q_{D}^{T}\right)$ be the transposes of these tableaux. (In the terminology of [42], this is EdelmanGreene "column insertion".)

(4) Let $I_{D}=\mathbf{i}_{D} \circ Q_{D}^{T}$ be the tableau with the same shape as $Q_{D}^{T}$ in which the entry $t$ replaced with $i_{t}$, for each $t=1, \ldots, p$. By Lenart's bijection [30, Remark 4.12(2)] (see also [42, Theorem 3.3]), the map $D \mapsto I_{D}$ is a weight preserving bijection from reduced pipe dreams for $\sigma_{\lambda}$ to column strict tableaux of shape $\lambda$ with row bounds $(1+x, 2+x, 3+x, \ldots)$. Call this family of $x$ flagged tableaux $\mathcal{F T}(\lambda, x)$. (The terminology of flagged tableaux is related to flagged Schur functions, see [47].)

(5) From the $x$-flagged tableau $I_{D}$, construct the filling $K_{D}$ by subtracting $u$ from every entry in row $u$. Note that the rows and columns are weakly increasing in $K_{D}$ and every entry is in the interval $[0, x]$, so $K_{D}$ is a reverse plane partition. Serrano and Stump prove in [41] that this is the bijection used by Fomin and Kirillov in Theorem 7.2 for the second equality.

The resulting map $F K:(\mathbf{a}, \mathbf{b}) \rightarrow\left(\mathbf{c}, K_{D}\right)$ is a bijection since each step is a bijection. It remains only to show that the $\mathfrak{q}$-weight is preserved. This follows from our bijective proof of Theorem 1.2 and the fact that specializing $x_{i}$ to $\mathfrak{q}^{i-1}$ in the Schubert polynomial specializes $x^{T}$ to $\mathfrak{q}^{b(\lambda)} \mathfrak{q}^{\left|K_{D}\right|}$.

\section{Future Directions}

We briefly mention some related open problems and connections to the literature here. Recall that pipe dreams can be encoded as bounded pairs, but most bounded pairs do not encode pipe dreams. In fact, in Section 3, we gave a simple test for this in terms of lexicographic order on certain related pairs. Perhaps there is another statistic 
based on these pairs which could be added to Macdonald's formula to find another generalization.

Open Problem 8.1. Is there an analog of the bounded pair polynomial in Definition 4.1 which specializes to the Schubert polynomial when certain parameters are set to 0? Is there a common generalization for the Transition Equation for Schubert polynomials, bounded pairs, and its $\mathfrak{q}$-analog?

Proctor's formula for plane partitions of staircase shape has a particularly nice factored form. This was key to the elegant formula in (13). Can the staircase shape be replaced, in any sense, with a more general partition $\lambda$ ?

In some sense the answer is "no". There exist rather general determinantal formulas for the partition function of the dimer model on a planar bipartite graph [19], and for ensembles of nonintersecting lattice paths in a directed acyclic graph [14]; it is famously possible to apply either formula to yield a determinantal formula for reverse plane partitions of arbitrary shapes (see, for instance, the book [4] for an introduction to this approach to plane partition enumeration). As observed in [11], typically this determinant cannot be written as a product of nice factors. Nonetheless, the more general Fomin-Kirillov formula in Theorem 7.2 makes it desirable to improve these enumerative results as much as possible.

Open Problem 8.2. Is there a nice formula for $\left|r p p^{\lambda}(x)\right|$ or $\left[r p p^{\lambda}(x)\right]_{\mathfrak{q}}$ for any large class of partitions $\lambda$, as in the case of staircase shapes as noted in Theorem 7.1?

Stembridge [46, Theorem 1.1] gives a formula for a weighted enumeration of maximal saturated chains in the Bruhat order for any Weyl group which is very similar to Macdonald's formula. This formula is related to the study of degrees of Schubert varieties, see [6,37], and has no obvious direct connection to Theorem 1.1. Stanley [45, Equation (23)] stated the following version of Stembridge's weighted enumeration formula in the case of $S_{n}$ and noted the similarity to Macdonald's formula. Given $w \in S_{n}$ of length $p$, let

$$
\begin{gathered}
\mathcal{T}(w):=\left\{\left(\left(i_{1}, j_{1}\right),\left(i_{2}, j_{2}\right), \ldots,\left(i_{p}, j_{p}\right)\right): w=t_{i_{1}, j_{1}} t_{i_{2}, j_{2}} \cdots t_{i_{p}, j_{p}}\right. \\
\text { and } \left.\ell\left(t_{i_{1}, j_{1}} t_{i_{2}, j_{2}} \cdots t_{i_{k}, j_{k}}\right)=k \text { for all } 1 \leqslant k \leqslant p\right\} .
\end{gathered}
$$

THEOREM 8.3. For $w=w_{0} \in S_{n}$, we have

$$
\sum_{\left(\left(i_{1}, j_{1}\right),\left(i_{2}, j_{2}\right), \ldots,\left(i_{p}, j_{p}\right)\right) \in \mathcal{T}\left(w_{0}\right)}\left(j_{1}-i_{1}\right)\left(j_{2}-i_{2}\right) \cdots\left(j_{p}-i_{p}\right)=\left(\begin{array}{l}
n \\
2
\end{array}\right) !
$$

Open Problem 8.4. Can (17) be proven bijectively, using a similar technique to our $M$ bijection?

The left side of (17) has a natural interpretation in terms of pairs $(\mathbf{a}, \mathbf{b})$ where $\mathbf{a}$ is a word of transpositions $\left(i_{k}, j_{k}\right)$, and $\mathbf{b}=\left(b_{1}, \ldots, b_{p}\right)$ is a sort of "bounded word" with bounds $i_{k} \leqslant b_{k}<j_{k}$. However, no analogue of Little's bumping map is known for maximal saturated Bruhat chains. Worse, (17) is only known to hold for the longest word $w_{0}$ and a few special cases. It would be necessary to find a generalization of (17) to other $w$ before the strategy outlined in this paper could apply.

The Grothendieck polynomials are the $K$-theory analog of Schubert polynomials for the flag manifolds [28]. There is a Transition Formula for these polynomials [26]. Anders Buch asked the following question.

Open Problem 8.5. What is the analog of Macdonald's formula for Grothendieck polynomials and what is the corresponding bijection? 
Fomin-Kirillov [9] state a Macdonald-type formula for the longest word $w_{0}$, as a corollary of their work on the degenerate Hecke algebra. Curiously the Stirling numbers of the second kind appear on the right side of the formula. There are also some partial recent partial results on this open problem due to Reiner, Tenner and Yong [40]. In particular, see their Definition 6.2 and Conjecture 6.3.

\section{ApPENDix}

For $\mathbf{a}=(4)$ with comaj $(\mathbf{a})=0$ and $j=5$, we have $v^{5}(\mathbf{a})=(4,3)$.

\begin{tabular}{|c|c|c|c|c|c|c|}
\hline$i$ & $h_{i}^{5}(\mathbf{a})$ & insert & $y_{i}^{5}(\mathbf{a})$ & $\operatorname{comaj}\left(y_{i}^{5}(\mathbf{a})\right)$ & $h_{i}^{5}(\mathbf{a})-1$ & $v_{i}^{5}(\mathbf{a})$ \\
\hline 0 & 5 & $(44)$ & $(54)$ & 0 & 4 & 4 \\
1 & 4 & $(43)$ & $(54)$ & 0 & 3 & 3 \\
\hline
\end{tabular}

For $\mathbf{a}=(4,3)$ with $\operatorname{comaj}(\mathbf{a})=0$ and $j=5$, we have $v^{5}(\mathbf{a})=(4,3,2)$.

\begin{tabular}{|c|c|c|c|c|c|c|}
\hline$i$ & $h_{i}^{5}(\mathbf{a})$ & insert & $y_{i}^{5}(\mathbf{a})$ & $\operatorname{comaj}\left(y_{i}^{5}(\mathbf{a})\right)$ & $h_{i}^{5}(\mathbf{a})-1$ & $v_{i}^{5}(\mathbf{a})$ \\
\hline 0 & 5 & $(443)$ & $(543)$ & 0 & 4 & 4 \\
1 & 4 & $(433)$ & $(543)$ & 0 & 3 & 3 \\
2 & 3 & $(432)$ & $(543)$ & 0 & 2 & 2 \\
\hline
\end{tabular}

For $\mathbf{a}=(4,3,5)$ with $\operatorname{comaj}(\mathbf{a})=2$ and $j=5$, we have $v^{5}(\mathbf{a})=(5,4,3,2)$.

\begin{tabular}{|c|c|c|c|c|c|c|}
\hline$i$ & $h_{i}^{5}(\mathbf{a})$ & insert & $y_{i}^{5}(\mathbf{a})$ & $\operatorname{comaj}\left(y_{i}^{5}(\mathbf{a})\right)$ & $h_{i}^{5}(\mathbf{a})-1$ & $v_{i}^{5}(\mathbf{a})$ \\
\hline 0 & 5 & $(4435)$ & $(5435)$ & 3 & 4 & 5 \\
1 & 4 & $(4335)$ & $(5435)$ & 3 & 3 & 4 \\
2 & 3 & $(4325)$ & $(5435)$ & 3 & 2 & 3 \\
3 & 3 & $(4352)$ & $(5453)$ & 2 & 2 & 2 \\
\hline
\end{tabular}

For $\mathbf{a}=(4,3,5,6)$ with $\operatorname{comaj}(\mathbf{a})=5$ and $j=5$, we have $v^{5}(\mathbf{a})=(6,5,4,3,2)$.

\begin{tabular}{|c|c|c|c|c|c|c|}
\hline$i$ & $h_{i}^{5}(\mathbf{a})$ & insert & $y_{i}^{5}(\mathbf{a})$ & $\operatorname{comaj}\left(y_{i}^{5}(\mathbf{a})\right)$ & $h_{i}^{5}(\mathbf{a})-1$ & $v_{i}^{5}(\mathbf{a})$ \\
\hline 0 & 5 & $(44356)$ & $(54356)$ & 7 & 4 & 6 \\
1 & 4 & $(43356)$ & $(54356)$ & 7 & 3 & 5 \\
2 & 3 & $(43256)$ & $(54356)$ & 7 & 2 & 4 \\
3 & 3 & $(43526)$ & $(54536)$ & 6 & 2 & 3 \\
4 & 3 & $(43562)$ & $(54563)$ & 5 & 2 & 2 \\
\hline
\end{tabular}

For $\mathbf{a}=(4,3,5,6,4)$ with $\operatorname{comaj}(\mathbf{a})=5$ and $j=5$, we have $v^{5}(\mathbf{a})=(6,5,4,3,7,2)$.

\begin{tabular}{|c|c|c|c|c|c|c|}
\hline$i$ & $h_{i}^{5}(\mathbf{a})$ & insert & $y_{i}^{5}(\mathbf{a})$ & $\operatorname{comaj}\left(y_{i}^{5}(\mathbf{a})\right)$ & $h_{i}^{5}(\mathbf{a})-1$ & $v_{i}^{5}(\mathbf{a})$ \\
\hline 0 & 5 & $(443564)$ & $(543564)$ & 7 & 4 & 6 \\
1 & 4 & $(433564)$ & $(543564)$ & 7 & 3 & 5 \\
2 & 3 & $(432564)$ & $(543564)$ & 7 & 2 & 4 \\
3 & 3 & $(435264)$ & $(545364)$ & 6 & 2 & 3 \\
4 & 3 & $(435624)$ & $(545634)$ & 10 & 2 & 7 \\
5 & 3 & $(435642)$ & $(435643)$ & 5 & 2 & 2 \\
\hline
\end{tabular}


For $\mathbf{a}=(4,3,5,6,4,3)$ with $\operatorname{comaj}(\mathbf{a})=5$ and $j=5$, we have $v^{5}(\mathbf{a})=$ $(6,5,4,3,7,8,9)$.

\begin{tabular}{|c|c|c|c|c|c|c|}
\hline$i$ & $h_{i}^{5}(\mathbf{a})$ & insert & $y_{i}^{5}(\mathbf{a})$ & $\operatorname{comaj}\left(y_{i}^{5}(\mathbf{a})\right)$ & $h_{i}^{5}(\mathbf{a})-1$ & $v_{i}^{5}(\mathbf{a})$ \\
\hline 0 & 5 & $(4435643)$ & $(5435654)$ & 7 & 4 & 6 \\
1 & 4 & $(4335643)$ & $(5435654)$ & 7 & 3 & 5 \\
2 & 3 & $(4325643)$ & $(5435654)$ & 7 & 2 & 4 \\
3 & 3 & $(4352643)$ & $(5453654)$ & 6 & 2 & 3 \\
4 & 3 & $(4356243)$ & $(5456354)$ & 10 & 2 & 7 \\
5 & 3 & $(4356423)$ & $(5456534)$ & 11 & 2 & 8 \\
6 & 4 & $(4356433)$ & $(5456534)$ & 11 & 3 & 9 \\
\hline
\end{tabular}

For $\mathbf{a}=(4,3,5,6,4,3,5)$ with $\operatorname{comaj}(\mathbf{a})=11$ and $j=5$, we have $v^{5}(\mathbf{a})=$ $(7,6,5,4,8,9,10,3)$.

\begin{tabular}{|c|c|c|c|c|c|c|}
\hline$i$ & $h_{i}^{5}(\mathbf{a})$ & insert & $y_{i}^{5}(\mathbf{a})$ & $\operatorname{comaj}\left(y_{i}^{5}(\mathbf{a})\right)$ & $h_{i}^{5}(\mathbf{a})-1$ & $v_{i}^{5}(\mathbf{a})$ \\
\hline 0 & 5 & $(44356435)$ & $(54356545)$ & 14 & 4 & 7 \\
1 & 4 & $(43356435)$ & $(54356545)$ & 14 & 3 & 6 \\
2 & 3 & $(43256435)$ & $(54356545)$ & 14 & 2 & 5 \\
3 & 3 & $(43526435)$ & $(54536545)$ & 13 & 2 & 4 \\
4 & 3 & $(43562435)$ & $(54563545)$ & 17 & 2 & 8 \\
5 & 3 & $(43564235)$ & $(54565345)$ & 18 & 2 & 9 \\
6 & 4 & $(43564335)$ & $(54565345)$ & 18 & 3 & 10 \\
7 & 4 & $(43564353)$ & $(43564354)$ & 11 & 3 & 3 \\
\hline
\end{tabular}

Acknowledgements. Many thanks to Connor Ahlbach, Sami Assaf, Anders Buch, Sergey Fomin, Ira Gessel, Zachary Hamaker, Avi Levy, Peter McNamara, Maria Monks Gillespie, Alejandro Morales, Richard Stanley, Dennis Stanton, Joshua Swanson, and Marisa Viola for helpful discussions on this work.

\section{REFERENCES}

[1] Nantel Bergeron and Sara C. Billey, RC-graphs and Schubert polynomials, Exp. Math. 2 (1993), no. $4,257-269$.

[2] Sara C. Billey, Zachary Hamaker, Austin Roberts, and Benjamin Young, Coxeter-Knuth graphs and a signed Little map for type B reduced words, Electron. J. Comb. 21 (2014), no. 4, P4.6 (39 pages).

[3] Sara C. Billey, William Jockusch, and Richard P. Stanley, Some combinatorial properties of Schubert polynomials, J. Algebr. Comb. 2 (1993), no. 4, 345-374.

[4] David M. Bressoud, Proofs and Confirmations: The Story of the Alternating-Sign Matrix Conjecture, Spectrum Series, Cambridge University Press, 1999.

[5] Leonard Carlitz, A combinatorial property of q-Eulerian numbers, Am. Math. Mon. 82 (1975) $51-54$.

[6] Claude Chevalley, Sur les décompositions cellulaires des espaces G/B, in Algebraic Groups and their generalizations: Classical methods (University Park, PA, 1991), Proceedings of Symposia in Pure Mathematics, vol. 56, Part 1, American Mathematical Society, 1994, pp. 1-23.

[7] Paul Edelman and Curtis Greene, Balanced tableaux, Adv. Math. 63 (1987), no. 1, 42-99.

[8] Dominique Foata, On the Netto inversion number of a sequence, Proc. Am. Math. Soc. 19 (1968), 236-240

[9] Sergey Fomin and Anatol N. Kirillov, Grothendieck polynomials and the Yang-Baxter equation, Proceedings of the Sixth Conference in Formal Power Series and Algebraic Combinatorics, Series in Discrete Mathematics and Theoretical Computer Science, American Mathematical Society, 1994, pp. 183-190.

[10] _ Yang-Baxter equation, symmetric functions, and Schubert polynomials, Discrete Math. 153 (1996), no. 1-3, 123-143.

[11] _ Reduced words and plane partitions, J. Algebr. Comb. 6 (1997), no. 4, 311-319.

[12] Sergey Fomin and Richard P. Stanley, Schubert polynomials and the nilCoxeter algebra, Adv. Math. 103 (1994), no. 2, 196-207. 
[13] Adriano Garsia, The Saga of Reduced Factorizations of Elements of the Symmetric Group, Publications du Laboratoire de Combinatoire et d'Informatique Mathématique, vol. 29, Laboratoire de combinatoire et d'informatique mathématique, 2002.

[14] Ira M. Gessel and Xavier Viennot, Determinants, paths, and plane partitions, manuscript, 1989.

[15] Hansraj Gupta, A new look at the permutations of the first $n$ natural numbers, Indian J. Pure Appl. Math. 9 (1978), no. 6, 600-631.

[16] James Haglund, Nicholas Loehr, and Jeffrey B. Remmel, Statistics on wreath products, perfect matchings, and signed words, Eur. J. Comb. 26 (2005), no. 6, 835-868.

[17] Zachary Hamaker, Eric Marberg, and Brendan Pawlowski, Transition formulas for involution Schubert polynomials, https://arxiv.org/abs/1609.09625, 2016.

[18] Zachary Hamaker and Benjamin Young, Relating Edelman-Greene insertion to the Little map, J. Algebr. Comb. 40 (2014), no. 3, 693-710.

[19] Pieter W. Kasteleyn, The statistics of dimers on a lattice: I. The number of dimer arrangements on a quadratic lattice, Physica 27 (1961), no. 12, 1209-1225.

[20] Allen Knutson, Schubert polynomials and symmetric functions; notes for the Lisbon combinatorics summer school 2012, 2012, http://www.math.cornell.edu/ allenk/schubnotes.pdf.

[21] Allen Knutson and Ezra Miller, Gröbner geometry of Schubert polynomials, Ann. Math. 161 (2005), no. 3, 1245-1318.

[22] Mikhail Kogan, Schubert geometry of flag varieties and Gelfand-Cetlin theory, Ph.D. thesis, Massachusetts Institute of Technology, 2000.

[23] Kazuhiko Koike and Itaru Terada, Young-diagrammatic methods for the representation theory of the classical groups of type $B_{n}, C_{n}, D_{n}$, J. Algebra 107 (1987), no. 2, 466-511.

[24] Thomas Lam, Luc Lapointe, Jennifer Morse, Anne Schilling, Mark Shimozono, and Mike Zabrocki, $k$-Schur Functions and Affine Schubert Calculus, Fields Institute Monographs, vol. 33, The Fields Institute for Research in the Mathematical Sciences, 2014.

[25] Thomas Lam and Mark Shimozono, A Little bijection for affine Stanley symmetric functions, Sémin. Lothar. Comb. 54A (2005), B54Ai (12 pages).

[26] Alain Lascoux, Transition on Grothendieck polynomials, in Proceedings of the Nagoya 2000 International Workshop, Physics and Combinatorics, World Scientific, 2000, pp. 164-179.

[27] Alain Lascoux and Marcel-Paul Schützenberger, Polynômes de Schubert, C. R. Acad. Sci., Paris, Sér. I 294 (1982), 447-450.

[28] _ Symmetry and flag manifolds, in Invariant theory (Montecatini, 1982), Lecture Notes in Mathematics, vol. 996, Springer, 1983, pp. 118-144.

[29] - Schubert polynomials and the Littlewood-Richardson rule, Lett. Math. Phys. 10 (1985), 111-124.

[30] Cristian Lenart, A unified approach to combinatorial formulas for Schubert polynomials, J. Algebr. Comb. 20 (2004), no. 3, 263-299.

[31] David P. Little, Combinatorial aspects of the Lascoux-Schützenberger tree, Adv. Math. 174 (2003), no. 2, 236-253.

[32] , Factorization of the Robinson-Schensted-Knuth correspondence, J. Comb. Theory, Ser. A 110 (2005), no. 1, 147-168.

[33] Ian G. Macdonald, Notes on Schubert Polynomials, Publications du Laboratoire de combinatoire et d'informatique mathématique, vol. 6, Université du Québec, 1991.

[34] Laurent Manivel, Symmetric Functions, Schubert Polynomials and Degeneracy Loci, SMF/AMS Texts and Monographs, vol. 6, American Mathematical Society, 2001.

[35] Maria Monks Gillespie, A combinatorial approach to the q,t-symmetry relation in Macdonald polynomials, Electron. J. Comb. 23 (2016), no. 2, P2.38 (64 pages).

[36] Mordechai Novick, A bijective proof of a major index theorem of Garsia and Gessel, Electron. J. Comb. 17 (2010), no. 1, 64 (12 pages).

[37] Alexander Postnikov and Richard P. Stanley, Chains in the Bruhat order, J. Algebr. Comb. 29 (2009), no. 2, 133-174.

[38] Robert A. Proctor, Odd symplectic groups, Invent. Math. 92 (1988), no. 2, 307-332.

[39] _ _ New symmetric plane partition identities from invariant theory work of De Concini and Procesi, Eur. J. Comb. 11 (1990), no. 3, 289-300.

[40] Victor Reiner, Bridget Eileen Tenner, and Alexander Yong, Poset edge densities, nearly reduced words, and barely set-valued tableaux, https://arxiv.org/abs/1603.09589, 2016.

[41] Luis Serrano and Christian Stump, Generalized triangulations, pipe dreams, and simplicial spheres, in 23rd International Conference on Formal Power Series and Algebraic Combinatorics (FPSAC 2011), Discrete Mathematics and Theoretical Computer Science, The Association. Discrete Mathematics \& Theoretical Computer Science (DMTCS), 2011, pp. 885-896. 
[42] - Maximal fillings of moon polyominoes, simplicial complexes, and Schubert polynomials, Electron. J. Comb. 19 (2012), no. 1, P16 (18 pages).

[43] Mark Skandera, An Eulerian partner for inversions, Sémin. Lothar. Comb. 46 (2001), B46d (19 pages).

[44] Richard P. Stanley, On the number of reduced decompositions of elements of Coxeter groups, Eur. J. Comb. 5 (1984), 359-372.

[45] - Permutations, 2009, http://www-math.mit.edu/ rstan/papers/perms.pdf.

[46] John R. Stembridge, A weighted enumeration of maximal chains in the Bruhat order, J. Algebr. Comb. 15 (2002), no. 3, 291-301.

[47] Michelle L. Wachs, Flagged Schur functions, Schubert polynomials, and symmetrizing operators, J. Comb. Theory, Ser. A 40 (1985), no. 2, 276-289.

[48] Anna Weigandt and Alexander Yong, The prism tableau model for Schubert polynomials, J. Comb. Theory, Ser. A 154 (2018), 551-582.

[49] Benjamin Young, A Markov growth process for Macdonald's distribution on reduced words, https://arxiv.org/abs/1409.7714, 2014.

Sara C. Billey, Department of Mathematics, University of Washington, Box 354350, Seattle, WA 98195, USA

E-mail : billey@math.washington.edu

Alexander E. Holroyd, Microsoft Research, 1 Microsoft Way, Redmond, WA 98052, USA

E-mail : holroyd@math.ubc.com

Benjamin J. Young, Department of Mathematics, 1222 University of Oregon, Eugene, OR 97403, USA

E-mail : bjy@uoregon.edu 